\title{
On the Eigenvalues of the Chandrasekhar-Page Angular Equation
}

\author{
Davide Batid* and Harald Schmid \\ NWF I - Mathematik, Universität Regensburg, D-93040 Regensburg, Germany. \\ Monika Winklmeielf \\ FB 3-Mathematik, Universität Bremen, D-28359 Bremen, Germany.
}

\begin{abstract}
In this paper we study for a given azimuthal quantum number $\kappa$ the eigenvalues of the Chandrasekhar-Page angular equation with respect to the parameters $\mu:=a m$ and $\nu:=a \omega$, where $a$ is the angular momentum per unit mass of a black hole, $m$ is the rest mass of the Dirac particle and $\omega$ is the energy of the particle (as measured at infinity). For this purpose, a self-adjoint holomorphic operator family $A(\kappa ; \mu, \nu)$ associated to this eigenvalue problem is considered. At first we prove that for fixed $\kappa \in \mathbb{R} \backslash\left(-\frac{1}{2}, \frac{1}{2}\right)$ the spectrum of $A(\kappa ; \mu, \nu)$ is discrete and that its eigenvalues depend analytically on $(\mu, \nu) \in \mathbb{C}^{2}$. Moreover, it will be shown that the eigenvalues satisfy a first order partial differential equation with respect to $\mu$ and $\nu$, whose characteristic equations can be reduced to a Painlevé III equation. In addition, we derive a power series expansion for the eigenvalues in terms of $\nu-\mu$ and $\nu+\mu$, and we give a recurrence relation for their coefficients. Further, it will be proved that for fixed $(\mu, \nu) \in \mathbb{C}^{2}$ the eigenvalues of $A(\kappa ; \mu, \nu)$ are the zeros of a holomorphic function $\Theta$ which is defined by a relatively simple limit formula. Finally, we discuss the problem if there exists a closed expression for the eigenvalues of the Chandrasekhar-Page angular equation.
\end{abstract}

\section{INTRODUCTION}

The angular eigenvalue problem of a spin- $\frac{1}{2}$ particle in the Kerr-Newman geometry is given by the ChandrasekharPage angular equation (see [3, Chap. 10, Sec. 104])

$$
\begin{aligned}
& \mathcal{L}_{\frac{1}{2}}^{+} S_{+\frac{1}{2}}=(a m \cos \theta-\lambda) S_{-\frac{1}{2}}, \\
& \mathcal{L}_{\frac{1}{2}}^{-} S_{-\frac{1}{2}}=(a m \cos \theta+\lambda) S_{+\frac{1}{2}},
\end{aligned}
$$

where the Kerr parameter $a$ is the angular momentum per unit mass of a black hole and $m$ is the rest mass of the Dirac particle. Moreover, the differential operators $\mathcal{L}_{\frac{1}{2}}^{ \pm}$are defined by

$$
\mathcal{L}_{\frac{1}{2}}^{ \pm}=\partial_{\theta} \pm Q(\theta)+\frac{\cot \theta}{2}, \quad Q(\theta):=a \omega \sin \theta+\frac{\kappa}{\sin \theta}, \quad \theta \in(0, \pi),
$$

where $\omega$ is the energy of the particle (as measured at infinity) and $\kappa$ is a half-integer, i.e., $\kappa=k-\frac{1}{2}$ with some $k \in \mathbb{Z}$. A parameter $\lambda \in \mathbb{R}$ is called an eigenvalue of this spectral problem if the system given by (11) - (2) has a nontrivial solution which is square-integrable on $(0, \pi)$ with respect to the weight function $\sin \theta$. In this paper we study for fixed $\kappa$ the eigenvalues of the Chandrasekhar-Page angular equation as a function of the parameters $\mu:=a m$ and $\nu:=a \omega$. As a main result, we will prove that the eigenvalues satisfy a first order quasi-linear partial differential equation, and we will derive a power series expansion for the eigenvalues in terms of $\nu-\mu$ and $\nu+\mu$.

For this purpose it is necessary to consider the system (11) - (2) in a more general context where $\kappa$ is real, $|\kappa| \geq \frac{1}{2}$, and $\mu, \nu$ are complex numbers. At first we rewrite this system for fixed $\kappa \in \mathbb{R} \backslash\left(-\frac{1}{2}, \frac{1}{2}\right)$ as an eigenvalue problem for some self-adjoint holomorphic operator family $A=A(\kappa ; \mu, \nu)$ depending on the parameters $(\mu, \nu) \in \mathbb{C}^{2}$. In the special case where $(\mu, \nu) \in \mathbb{R}^{2}$ the differential operator $A(\kappa ; \mu, \nu)$ is self-adjoint and has purely discrete spectrum. In Section II we prove that for a given $\kappa$ the eigenvalues $\lambda_{j}(\kappa ; \mu, \nu)$ of $A$ are holomorphic functions in $(\mu, \nu)$, and we derive some basic estimates for them. Furthermore, we transform the system (11) - (2) to a matrix differential equation

$$
y^{\prime}(x)=\left[\frac{1}{x} B_{0}+\frac{1}{x-1} B_{1}+C\right] y(x)
$$

\footnotetext{
*Electronic address: davide.batic@mathematik.uni-regensburg.de

$\dagger$ Electronic address: harald.schmid@mathematik.uni-regensburg.de

‡Electronic address: winklmeier@math.uni-bremen.de
} 
on the interval $(0,1)$ with coefficient matrices

$$
B_{0}=\left(\begin{array}{cc}
-\frac{\kappa}{2}-\frac{1}{4} & \mu-\lambda \\
0 & \frac{\kappa}{2}+\frac{1}{4}
\end{array}\right), \quad B_{1}=\left(\begin{array}{cc}
\frac{\kappa}{2}+\frac{1}{4} & 0 \\
\mu-\lambda & -\frac{\kappa}{2}-\frac{1}{4}
\end{array}\right), \quad C=\left(\begin{array}{cc}
-2 \nu & -2 \mu \\
2 \mu & 2 \nu
\end{array}\right),
$$

which can be extended to the complex domain $\mathbb{C} \backslash\{0,1\}$. In this way we obtain a further characterisation of the eigenvalues of $A$ and some useful estimates for the corresponding eigenfunctions. Applying analytic perturbation theory, we show in Section III that the eigenvalues $\lambda_{j}(\kappa ; \mu, \nu)$ satisfy the partial differential equation

$$
(\mu-2 \nu \lambda) \frac{\partial \lambda}{\partial \mu}+(\nu-2 \mu \lambda) \frac{\partial \lambda}{\partial \nu}+2 \kappa \mu+2 \mu \nu=0 .
$$

In particular, this result can be used to obtain a recurrence relation for the coefficients $c_{m, n}$ of a power series expansion

$$
\lambda_{j}(\kappa ; \mu, \nu)=\sum_{m, n=0}^{\infty} c_{m, n}(\nu-\mu)^{m}(\nu+\mu)^{n} .
$$

In Section IV we solve the PDE (4) by the method of characteristics. First, we derive an explicit formula for the eigenvalues in the case $|\mu|=|\nu|$. Moreover, in the regions where $|\mu| \neq|\nu|$ we reduce the characteristic equations of (44) to a Painlevé III equation

$$
v v^{\prime}+t v v^{\prime \prime}-t\left(v^{\prime}\right)^{2}-2 \kappa\left(v^{2} \pm 1\right) v-t\left(v^{4}-1\right)=0
$$

with parameters $\alpha= \pm \beta=2 \kappa$ and $\gamma=-\delta=1$ according to the notation in [18] and [16]. As this differential equation is in general not solvable in terms of elementary functions, we cannot expect a closed expression for the eigenvalues of the Chandrasekhar-Page angular equation for all $(\mu, \nu) \in \mathbb{R}^{2}$. However, if $\kappa$ is a half-integer, i.e., $\kappa=k-\frac{1}{2}$ with some positive integer $k$, then $\alpha \pm \beta=2(2 k-1)$, and there are integrals of polynomial type for the third Painlevé equation in this special case, cf. [18]. Hence, if $\kappa= \pm \frac{1}{2}, \pm \frac{3}{2}, \ldots$, there exist algebraic solutions of the partial differential equation (44), and the question arises if these explicit solutions are in fact eigenvalues of the Chandrasekhar-Page angular equation. It turns out that there is another type of "special values" associated to the operator $A$, called monodromy eigenvalues, which belong to the algebraic solutions of the PDE (4). For a half-integer $\kappa$, the monodromy eigenvalues are introduced in Section $\mathrm{V}$ by requiring that the system (3) has a fundamental matrix of the form

$$
[x(1-x)]^{-\frac{\kappa}{2}-\frac{1}{4}} H(x)
$$

with an entire matrix function $H: \mathbb{C} \longrightarrow \mathrm{M}_{2}(\mathbb{C})$. This property turns out to be equivalent to the existence of special solutions of the form

$$
[x(1-x)]^{-\frac{\kappa}{2}-\frac{1}{4}} p^{ \pm}(x) e^{ \pm 2 t x},
$$

where $p^{ \pm}: \mathbb{C} \longrightarrow \mathbb{C}^{2}$ are polynomials and $t= \pm \sqrt{\nu^{2}-\mu^{2}}$. For comparison purposes, an eigenvalue of $A$ can be characterised by the property that (3) possesses a nontrivial solution of the form

$$
[x(1-x)]^{\frac{\kappa}{2}+\frac{1}{4}} \eta(x)
$$

with some entire vector function $\eta: \mathbb{C} \longrightarrow \mathbb{C}^{2}$. We prove that the monodromy eigenvalues are zeros of a polynomial with degree $2 k-1$ whose coefficients are polynomials in $\mu$ and $\nu$. Moreover, it can be shown that monodromy eigenvalues and "classical" eigenvalues are distinct at least in a neighbourhood of $(\mu, \nu)=(0,0)$. Nevertheless, they are both characterised by the fact that certain monodromy data of the system (3) are preserved for all parameters $(\mu, \nu)$. In fact, $\lambda$ is a monodromy eigenvalue of $A$ if and only if the monodromy matrices of (3) at the regular-singular points 0 and 1 are diagonal, whereas $\lambda$ is a classical eigenvalue of $A$ if and only if a certain non-diagonal entry of the connection matrix for the fundamental matrices at 0 and 1 vanishes. Hence, for the Chandrasekhar-Page angular equation the monodromy as well as the classical eigenvalue problem is closely related to the isomonodromy problem for the differential equation (3). Monodromy preserving deformations for such a system were studied by Jimbo, Miwa \& Ueno in [9], however, only for the case that the eigenvalues of $B_{0}$ and $B_{1}$ do not differ by an integer, i.e., $\kappa+\frac{1}{2} \notin \mathbb{Z}$. In Section VI we consider the isomonodromy problem for (3) in the case that $\kappa$ is a half-integer. As a consequence, we show that the monodromy eigenvalues of $A$ satisfy the partial differential equation (4), and we obtain an alternative derivation of (44) for the classical eigenvalues of $A$. Unlike the proof in Section III, which relies on the particular structure of the Chandrasekhar-Page angular equation, the method presented in Section V is more general and based on finding suitable deformation equations for parameter-dependent differential equations. Thus, we expect that this technique is applicable to other eigenvalue problems as well. 


\section{A SELF-ADJOINT HOLOMORPHIC OPERATOR FAMILY ASSOCIATED TO THE CHANDRASEKHAR-PAGE ANGULAR EQUATION}

By introducing the notations

$$
\mu:=a m, \quad \nu:=a \omega, \quad S(\theta):=\sqrt{\sin \theta}\left(\begin{array}{c}
S_{+\frac{1}{2}}(\theta) \\
S_{-\frac{1}{2}}(\theta)
\end{array}\right)
$$

the Chandrasekhar-Page angular equation (11) - (2) takes the form

$$
(\mathfrak{A} S)(\theta):=\left(\begin{array}{cc}
0 & 1 \\
-1 & 0
\end{array}\right) S^{\prime}(\theta)+\left(\begin{array}{cc}
-\mu \cos \theta & -\frac{\kappa}{\sin \theta}-\nu \sin \theta \\
-\frac{\kappa}{\sin \theta}-\nu \sin \theta & \mu \cos \theta
\end{array}\right) S(\theta)=\lambda S(\theta), \quad \theta \in(0, \pi)
$$

with fixed $\kappa \in \mathbb{R} \backslash\left(-\frac{1}{2}, \frac{1}{2}\right)$ and parameters $(\mu, \nu) \in \mathbb{C}^{2}$. We can associate the so called minimal operator $A_{0}$ to the formal differential expression $\mathfrak{A}$, which acts in the Hilbert space $\mathcal{H}:=\mathscr{L}^{2}\left((0, \pi), \mathbb{C}^{2}\right)$ of square integrable vector functions with respect to the scalar product

$$
\left(S_{1}, S_{2}\right):=\int_{0}^{\pi} S_{2}(\theta)^{*} S_{1}(\theta) \mathrm{d} \theta, \quad S_{1}, S_{2} \in \mathcal{H} .
$$

The operator $A_{0}$ given by $\mathcal{D}\left(A_{0}\right)=\mathrm{C}_{0}^{\infty}\left((0, \pi), \mathbb{C}^{2}\right)$ and $A_{0} S:=\mathfrak{A} S$ for $S \in \mathcal{D}\left(A_{0}\right)$ is densely defined and closable. For $|\kappa| \geq \frac{1}{2}$ and $(\mu, \nu) \in \mathbb{R}^{2}$ the formal differential operator in (5) is in the limit point case at 0 and $\pi$, hence $A_{0}$ is even essentially self-adjoint. In the following we denote the closure of $A_{0}$ by $A=A(\kappa ; \mu, \nu)$. According to $[25$, Theorem 5.8] the domain of $A(\kappa ; 0,0)$ is given by

$$
\mathcal{D}(A)=\{S \in \mathcal{H}: S \text { is absolutely continuous and } A(\kappa ; 0,0) S \in \mathcal{H}\}
$$

Since $A(\kappa ; \mu, \nu)=A(\kappa ; 0,0)+T(\mu, \nu)$ with the bounded multiplication operator

$$
T(\mu, \nu)=\left(\begin{array}{cc}
-\mu \cos \theta & -\nu \sin \theta \\
-\nu \sin \theta & \mu \cos \theta
\end{array}\right)
$$

its domain of definition $\mathcal{D}(A)$ is independent of $(\mu, \nu) \in \mathbb{C}^{2}$ (see 14, Chap. IV, $\S 1$, Theorem 1.1]). Moreover, if $(\mu, \nu) \in \mathbb{R}^{2}$, then $T(\mu, \nu)$ is a symmetric perturbation of $A(\kappa ; 0,0)$, and [14, Chap. V, $\S 4$, Theorem 4.10] yields that $A(\kappa ; \mu, \nu)$ is self-adjoint. Thus, according to Kato's classification [14, Chap. VII, $\S 3], A(\kappa ; \mu, \nu)$ forms a self-adjoint holomorphic operator family of type $(\mathrm{A})$ in the variables $(\mu, \nu) \in \mathbb{C}^{2}$. Further, the spectrum of $A(\kappa ; 0,0)$ is discrete and consists of simple eigenvalues given by

$$
\lambda_{j}(\kappa ; 0,0)=\operatorname{sgn}(j)\left(|\kappa|-\frac{1}{2}+|j|\right), \quad j \in \mathbb{Z} \backslash\{0\}
$$

(for the details we refer to Appendix A). This means, in particular, that $A(\kappa ; 0,0)$ has compact resolvent, and from 14, Chap. V, $\S 2$, Theorem 2.4] it follows that $A(\kappa ; \mu, \nu)$ has compact resolvent for all $(\mu, \nu) \in \mathbb{C}^{2}$. As a consequence, the spectrum of $A(\kappa ; \mu, \nu),(\mu, \nu) \in \mathbb{C}^{2}$, is discrete, and since $A(\kappa ; \mu, \nu)$ is in the limit point case at $\theta=0$ and $\theta=\pi$, it consists of simple eigenvalues for $(\mu, \nu) \in \mathbb{R}^{2}$. Now, 14, Chap. V, $\S 3$, Theorem 3.9] implies that the eigenvalues $\lambda_{j}=\lambda_{j}(\kappa ; \mu, \nu), j \in \mathbb{Z} \backslash\{0\}$, of $A(\kappa ; \mu, \nu)$ are simple and depend holomorphically on $(\mu, \nu)$ in a complex neighbourhood of $\mathbb{R}^{2}$. Moreover, the partial derivatives of $A$ with respect to $\mu$ and $\nu$ are given by

$$
\frac{\partial A}{\partial \mu}=\left(\begin{array}{cc}
-\cos \theta & 0 \\
0 & \cos \theta
\end{array}\right), \quad \frac{\partial A}{\partial \nu}=\left(\begin{array}{cc}
0 & -\sin \theta \\
-\sin \theta & 0
\end{array}\right),
$$

which yields the following estimates for the growth rate of the eigenvalues (compare [14, Chap. VII, §3, Sec. 4]):

$$
\left|\frac{\partial \lambda_{j}}{\partial \mu}\right| \leq\left\|\frac{\partial A}{\partial \mu}\right\| \leq 1, \quad\left|\frac{\partial \lambda_{j}}{\partial \nu}\right| \leq\left\|\frac{\partial A}{\partial \nu}\right\| \leq 1 .
$$

Here, $\|\cdot\|$ denotes the operator norm of a $(2 \times 2)$ matrix. In addition, by [14, Chap. V, $\S 3$, Theorem 4.10], we have

$$
\min _{j \in \mathbb{Z} \backslash\{0\}}\left|\lambda-\lambda_{j}(\kappa ; 0,0)\right| \leq\|T(\mu, \nu)\| \leq \max \{|\mu|,|\nu|\}
$$


for each eigenvalue $\lambda$ of $A(\kappa ; \mu, \nu)$. Finally, by interchanging the components of $S(\theta)$, we obtain that a point $\lambda$ is an eigenvalue of $A(\kappa ; \mu, \nu)$ if and only if $-\lambda$ is an eigenvalue of $A(-\kappa ; \mu,-\nu)$. Since the eigenvalues depend holomorphically on $\mu$ and $\nu$, the identity

$$
\lambda_{j}(\kappa ; \mu, \nu)=-\lambda_{-j}(-\kappa ; \mu,-\nu)
$$

holds for all $(\mu, \nu)$ in a neighbourhood of $\mathbb{R}^{2}$. Therefore, we restrict our attention to the case $\kappa \in\left[\frac{1}{2}, \infty\right)$. Note that $\lambda \in \mathbb{C}$ is an eigenvalue of $A(\kappa ; \mu, \nu)$ if and only if the system (5) has a nontrivial solution $S(\theta)$ satisfying

$$
\int_{0}^{\pi}|S(\theta)|^{2} \mathrm{~d} \theta<\infty
$$

By means of the transformation

$$
S(\theta)=\left(\begin{array}{cc}
\sqrt{\tan \frac{\theta}{2}} & 0 \\
0 & \sqrt{\cot \frac{\theta}{2}}
\end{array}\right) y\left(\sin ^{2} \frac{\theta}{2}\right), \quad \theta \in(0, \pi),
$$

the differential equation (5) is equivalent to the system

$$
y^{\prime}(x)=\left[\frac{1}{x} B_{0}+\frac{1}{x-1} B_{1}+C\right] y(x)
$$

on the interval $(0,1)$ with coefficient matrices

$$
B_{0}:=\left(\begin{array}{cc}
-\frac{\kappa}{2}-\frac{1}{4} & \mu-\lambda \\
0 & \frac{\kappa}{2}+\frac{1}{4}
\end{array}\right), \quad B_{1}:=\left(\begin{array}{cc}
\frac{\kappa}{2}+\frac{1}{4} & 0 \\
\mu-\lambda & -\frac{\kappa}{2}-\frac{1}{4}
\end{array}\right), \quad C:=\left(\begin{array}{cc}
-2 \nu & -2 \mu \\
2 \mu & 2 \nu
\end{array}\right),
$$

and the normalisation condition (9) becomes

$$
\int_{0}^{1} y(x)^{*}\left(\begin{array}{cc}
\frac{1}{1-x} & 0 \\
0 & \frac{1}{x}
\end{array}\right) y(x) \mathrm{d} x<\infty .
$$

If we consider the differential equation (11) for a fixed $\kappa \in(0, \infty)$ in the complex plane, then it has two regular singular points, one at $x=0$ and one at $x=1$ with characteristic values $\pm\left(\frac{\kappa}{2}+\frac{1}{4}\right)$. From the theory of asymptotic expansions (see [24], for example), it follows that for each $\lambda \in \mathbb{C}$ there exists a nontrivial solution

$$
y_{0}(x, \lambda)=x^{\frac{\kappa}{2}+\frac{1}{4}} h(x, \lambda), \quad x \in \mathfrak{B}_{0},
$$

of (11) in the unit disc $\mathfrak{B}_{0} \subset \mathbb{C}$ with centre 0 , where $h(\cdot, \lambda): \mathfrak{B}_{0} \longrightarrow \mathbb{C}^{2}$ is a holomorphic function,

$$
h(x, \lambda)=\sum_{n=0}^{\infty} x^{n} h_{n}(\lambda), \quad h_{0}(\lambda):=\left(\begin{array}{c}
\mu-\lambda \\
\kappa+\frac{1}{2}
\end{array}\right) .
$$

Here $h_{0}(\lambda)$ is an eigenvector of $B_{0}$ for the eigenvalue $\frac{\kappa}{2}+\frac{1}{4}$, and the coefficients $h_{n}(\lambda), n>1$, are uniquely determined by the recurrence relation

$$
\left(B_{0}-\alpha-n\right) h_{n}(\lambda)=\left(B_{0}+B_{1}-C+1-\alpha-n\right) h_{n-1}(\lambda)+C h_{n-2}(\lambda)
$$

with $\alpha:=\frac{\kappa}{2}+\frac{1}{4}$ and $h_{-1}(\lambda):=0$. Since the matrices $B_{0}$ and $B_{1}$ depend holomorphically on $\lambda$, the coefficients $h_{n}: \mathbb{C} \longrightarrow \mathbb{C}^{2}$ are holomorphic functions. By slightly modifying the proof of [24, Theorem 5.3], it can be shown that the series (15) converges uniformly in every compact subset of $\mathfrak{B}_{0} \times \mathbb{C}$. Thus, by a theorem of Weierstrass, $h: \mathfrak{B}_{0} \times \mathbb{C} \longrightarrow \mathbb{C}^{2}$ is a holomorphic vector function in the variables $(x, \lambda)$. Now, let

$$
h\left(\frac{1}{2}, \lambda\right)=:\left(\begin{array}{l}
f(\lambda) \\
g(\lambda)
\end{array}\right)
$$

and we define the holomorphic function $\Delta: \mathbb{C} \longrightarrow \mathbb{C}$ by

$$
\Delta(\lambda):=f(\lambda)^{2}-g(\lambda)^{2}, \quad \lambda \in \mathbb{C} .
$$

The following Lemma provides a connection between the eigenvalues of $A$ and the zeros of $\Delta$. 
Lemma 1 For fixed $\kappa \in\left[\frac{1}{2}, \infty\right)$ and $(\mu, \nu) \in \mathbb{C}^{2}$, a point $\lambda \in \mathbb{C}$ is an eigenvalue of $A(\kappa ; \mu, \nu)$ if and only if $\lambda$ is a zero of the function $\Delta$ given by (17). This is equivalent to the statement that the differential equation (11) has a nontrivial solution of the form

$$
y(x)=[x(1-x)]^{\frac{\kappa}{2}+\frac{1}{4}} \eta(x), \quad x \in \mathbb{C} \backslash\{0,1\},
$$

where $\eta: \mathbb{C} \longrightarrow \mathbb{C}^{2}$ is an entire vector function. As a consequence, if $S$ is an eigenfunction of $A(\kappa ; \mu, \nu)$ for some eigenvalue $\lambda$, then

$$
|S(\theta)| \leq C \sin ^{\kappa} \theta, \quad \theta \in(0, \pi)
$$

with some constant $C>0$.

Proof. Defining

$$
K:=\left(\begin{array}{ll}
0 & 1 \\
1 & 0
\end{array}\right)
$$

we have $K^{-1}=K$ and $K B_{0} K=B_{1}, K C K=-C$. Hence, $y$ is a solution of the system (II) if and only if the function $K y(1-x)$ satisfies (11). In particular, $y_{1}(x):=K y_{0}(1-x)$ is a solution of (11) in the unit disc $\mathfrak{B}_{1} \subset \mathbb{C}$ with centre 1 , and $y_{1}$ has the form

$$
y_{1}(x, \lambda)=(1-x)^{\frac{\kappa}{2}+\frac{1}{4}} K h(1-x, \lambda), \quad x \in \mathfrak{B}_{1} .
$$

Moreover, by the Levinson Theorem (see [5, Theorem 1.3.1]), any solution of (11) which is linearly independent of $y_{0}$ in $(0,1)$ behaves asymptotically like $x^{-\frac{\kappa}{2}-\frac{1}{4}}\left[v_{0}+o(1)\right]$ as $x \rightarrow 0$, where $v_{0}$ is an eigenvector of $B_{0}$ for the eigenvalue $-\frac{\kappa}{2}-\frac{1}{4}$. Similarly, any solution of (11) which is linearly independent of $y_{1}$ in $(0,1)$ has the asymptotic behaviour $(x-1)^{-\frac{\kappa}{2}-\frac{1}{4}}\left[v_{1}+o(1)\right]$ as $x \rightarrow 1$ with an eigenvector $v_{1}$ of $B_{1}$ for the eigenvalue $-\frac{\kappa}{2}-\frac{1}{4}$. Now, if $\lambda$ is an eigenvalue of $A(\kappa ; \mu, \nu)$, then the system (11) has a nontrivial solution $y$ satisfying (13), and it follows that $y(x)=y_{a}(x, \lambda) c_{a}$ holds in $(0,1)$ with some constants $c_{a} \in \mathbb{C} \backslash\{0\}, a \in\{0,1\}$. Thus, $y_{0}$ and $y_{1}$ are linearly dependent, and the Wronskian $W(x, \lambda):=\operatorname{det}\left(y_{0}(x, \lambda), y_{1}(x, \lambda)\right)$ vanishes identically for all $x \in(0,1)$. In particular, $0=W\left(\frac{1}{2}, \lambda\right)=2^{-\kappa-\frac{1}{2}} \Delta(\lambda)$. Conversely, if $\Delta(\lambda)=0$, then $W\left(\frac{1}{2}, \lambda\right)=0$, which implies that $y_{0}$ and $y_{1}$ are linearly dependent. Hence, $y_{0}(x)=y_{1}(x) c$ with some constant $c \in \mathbb{C} \backslash\{0\}$, and therefore $y_{0}$ is a solution of (11) satisfying the condition (13) on the interval $(0,1)$. Moreover, we immediately obtain that $y_{0}$ has the form (18) with a holomorphic vector function $\eta: \mathfrak{B}_{0} \cup \mathfrak{B}_{1} \longrightarrow \mathbb{C}^{2}$, and since (11) is regular in $\mathbb{C} \backslash\{0,1\}$, we can extend $\eta: \mathbb{C} \longrightarrow \mathbb{C}^{2}$ to an entire function by the existence and uniqueness theorem. Finally, by means of the transformation (10), an eigenfunction $S$ of $A(\kappa ; \mu, \nu)$ has to be a constant multiple of

$$
\sin ^{\kappa} \theta\left(\begin{array}{cc}
\sin \frac{\theta}{2} & 0 \\
0 & \cos \frac{\theta}{2}
\end{array}\right) \eta\left(\sin ^{2} \frac{\theta}{2}\right), \quad \theta \in(0, \pi),
$$

and this yields the estimate (19).

Lemma 2 For fixed $\kappa \in\left[\frac{1}{2}, \infty\right)$ and $j \in \mathbb{Z} \backslash\{0\}$, the $j$-th eigenvalue $\lambda_{j}(\kappa ; \mu, \nu)$ of $A(\kappa ; \mu, \nu)$ has a power series expansion of the form

$$
\lambda_{j}(\kappa ; \mu, \nu)=\sum_{m, n=0}^{\infty} \lambda_{m, n} \mu^{m} \nu^{n}, \quad \lambda_{0,0}=\lambda_{j}(\kappa ; 0,0),
$$

which is uniformly convergent in the polydisc $\mathfrak{C}:=\left\{(\mu, \nu) \in \mathbb{C}^{2}:|\mu|,|\nu| \leq \frac{1}{2}\right\}$. Moreover, for all integers $m$ and $n$, the following estimate holds:

$$
\left|\lambda_{m, n}\right| \leq(|\kappa|+|j|) 2^{n+m}
$$

Proof. Since the coefficient matrices in (11]) depend holomorphically on $(\lambda, \mu, \nu) \in \mathbb{C}^{3}$, we can modify 24 , Theorem 5.3] appropriately in order to obtain that $h$ in (14) and therefore $\Delta=\Delta(\lambda, \mu, \nu)$ as given by (17) are holomorphic functions on $\mathbb{C}^{3}$. By a similar reasoning as in the proof of Lemma 1 we can show that for fixed $(\mu, \nu) \in \mathbb{C}^{2}$ the eigenvalues of $A(\kappa ; \mu, \nu)$ coincide with the zeros of the function $\lambda \longmapsto \Delta(\lambda, \mu, \nu)$. In particular for the case 
$(\mu, \nu) \in \mathbb{R}^{2}$ these zeros are simple because $A(\kappa ; \mu, \nu)$ has only simple eigenvalues. Hence, by solving the equation $\Delta(\lambda, \mu, \nu)=0$ and using the implicit function theorem, an eigenvalue $\lambda_{j}(\kappa ; \mu, \nu)$ of the operator $A(\kappa ; \mu, \nu)$ depends holomorphically on $(\mu, \nu)$ in a complex neighbourhood of $\mathbb{R}^{2}$. Furthermore, the estimate (8) implies that the set $\left\{\lambda \in \mathbb{C}: \min _{j \neq 0}\left|\lambda-\lambda_{j}(\kappa ; 0,0)\right| \geq \frac{1}{2}\right\}$ contains no eigenvalues of $A(\kappa ; \mu, \nu)$ for all $(\mu, \nu) \in \mathfrak{C}$. Thus there exists a holomorphic solution $\lambda: \mathfrak{C} \longrightarrow \mathbb{C}$ of the equation $\Delta(\lambda, \mu, \nu)=0$, which is uniquely determined by $\lambda(0,0)=\lambda_{j}(\kappa ; 0,0)$. Consequently, $\lambda_{j}(\kappa ; \mu, \nu)$ is holomorphic in $\mathfrak{C}$, and therefore it has a power series expansion in $\mathfrak{C}$ of the form (21). In addition, by Cauchy's formula,

$$
\lambda_{m, n}=-\frac{1}{4 \pi^{2}} \oint_{\partial \mathfrak{C}} \frac{\lambda_{j}(\kappa ; \mu, \nu)}{\mu^{m+1} \nu^{n+1}} \mathrm{~d} \mu \mathrm{d} \nu
$$

and applying (8) and (7), it follows that

$$
\left|\lambda_{j}(\kappa ; \mu, \nu)\right| \leq\left|\lambda_{j}(\kappa ; 0,0)\right|+\max \{|\mu|,|\nu|\} \leq|\kappa|+|j|
$$

which gives the estimate (22).

According to Lemma \ for fixed parameters $(\mu, \nu) \in \mathbb{C}^{2}$ the eigenvalues of $A(\kappa ; \mu, \nu)$ are exactly the zeros of the function $\Delta(\lambda)$ given by (17). In principle, this result can be used for numerical computation of the eigenvalues. However, in order to calculate $\Delta(\lambda)$ at some point $\lambda \in \mathbb{C}$, we first have to determine the coefficients $h_{n}(\lambda)$ with the help of the recurrence relation (16) and subsequently we need to evaluate $h(x, \lambda)$ at $x=\frac{1}{2}$ by means of the power series expansion (15). Unfortunately, this method requires the calculation of two consecutive limits, making things rather complicated. In the remaining part of this section we show that there is yet another function $\Theta$ which encodes the eigenvalues of $A(\kappa ; \mu, \nu)$. The main advantage of $\Theta$ is, that it can be obtained by only one limit process.

By setting $y(x):=x^{\alpha}(1-x)^{1-\alpha} \hat{y}(x)$ with $\alpha:=\frac{\kappa}{2}+\frac{1}{4}$, the system (11) becomes

$$
\hat{y}^{\prime}(x)=\left[\frac{1}{x} \hat{B}_{0}+\frac{1}{x-1} \hat{B}_{1}+C\right] \hat{y}(x)
$$

with the coefficient matrices

$$
\hat{B}_{0}:=\left(\begin{array}{cc}
-\kappa-\frac{1}{2} & \mu-\lambda \\
0 & 0
\end{array}\right), \quad \hat{B}_{1}:=\left(\begin{array}{cc}
\kappa-\frac{1}{2} & 0 \\
\mu-\lambda & -1
\end{array}\right), \quad C=\left(\begin{array}{cc}
-2 \nu & -2 \mu \\
2 \mu & 2 \nu
\end{array}\right) .
$$

Now, there exists a holomorphic solution of $(23)$ in $\mathfrak{B}_{1}$ given by

$$
\hat{y}(x, \lambda)=\sum_{n=0}^{\infty} x^{n} d_{n}(\lambda), \quad d_{0}(\lambda):=\left(\begin{array}{c}
\mu-\lambda \\
\kappa+\frac{1}{2}
\end{array}\right),
$$

where $d_{0}(\lambda)$ is an eigenvector of $\hat{B}_{0}$ for the eigenvalue 0 . In addition, the coefficients $d_{n}(\lambda), n>1$, are uniquely determined by the recurrence relation

$$
d_{n}(\lambda)=\left(\hat{B}_{0}-n\right)^{-1}\left[(E-n) d_{n-1}(\lambda)+C d_{n-2}(\lambda)\right]
$$

with

$$
E:=\left(\begin{array}{cc}
2 \nu & 3 \mu-\lambda \\
-\mu-\lambda & -2 \nu
\end{array}\right), \quad d_{-1}(\lambda):=0 .
$$

Finally, we denote by $\Theta_{n}(\lambda)$ the second component of $d_{n}(\lambda)$.

Lemma 3 Let $\kappa \in\left[\frac{1}{2}, \infty\right)$ and $(\mu, \nu) \in \mathbb{C}^{2}$ be fixed. Then, for each $\lambda \in \mathbb{C}$, the limit

$$
\Theta(\lambda):=\lim _{n \rightarrow \infty} \Theta_{n}(\lambda)
$$

exists, and $\Theta: \mathbb{C} \longrightarrow \mathbb{C}$ is a holomorphic function. Moreover, a point $\lambda \in \mathbb{C}$ is an eigenvalue of $A(\kappa ; \mu, \nu)$ if and only if $\Theta(\lambda)=0$. 
Proof. For fixed $\lambda \in \mathbb{C}$, the differential equation (23) has a regular singular point at $x=1$ with characteristic values -1 and $\kappa-\frac{1}{2}$. First, let us assume that their difference $\kappa+\frac{1}{2}$ is not an integer. In this case the system (23) has a fundamental system of solutions in a complex neighbourhood of $x=1$, which can be written as

$$
\hat{y}_{1}(x, \lambda)=(1-x)^{-1} \sum_{n=0}^{\infty}(1-x)^{n} d_{n}^{1}(\lambda), \quad \hat{y}_{2}(x, \lambda)=(1-x)^{\kappa-\frac{1}{2}} \sum_{n=0}^{\infty}(1-x)^{n} d_{n}^{2}(\lambda),
$$

where

$$
d_{0}^{1}(\lambda)=\left(\begin{array}{l}
0 \\
1
\end{array}\right)=: e_{2}, \quad d_{0}^{2}(\lambda)=\left(\begin{array}{c}
\kappa+\frac{1}{2} \\
\mu-\lambda
\end{array}\right)
$$

are eigenvectors of $\hat{B}_{1}$ for the eigenvalues -1 and $\kappa-\frac{1}{2}$, respectively. Now, $\hat{y}$ can be written as a linear combination

$$
\hat{y}(x, \lambda)=\gamma_{1}(\lambda) \hat{y}_{1}(x, \lambda)+\gamma_{2}(\lambda) \hat{y}_{2}(x, \lambda)
$$

with connection coefficients $\gamma_{1}(\lambda), \gamma_{2}(\lambda) \in \mathbb{C}$. Applying [22, Corollary 1.6] to the system (23) gives

$$
\lim _{n \rightarrow \infty} d_{n}(\lambda)=\gamma_{1}(\lambda) e_{2}
$$

and therefore the limit (25) exists. Furthermore, $\lambda$ is an eigenvalue of $A(\kappa ; \mu, \nu)$ if and only if $\gamma_{1}(\lambda)=0$, i.e., if and only if $\Theta(\lambda)$ becomes zero. Finally, it can be shown that the functions $d_{n}$ converge uniformly in every compact subset of $\mathbb{C}$, and Weierstrass' theorem implies that $\Theta$ is an entire function.

Now, suppose that $k:=\kappa+\frac{1}{2}$ is a positive integer. In this case, a fundamental system of the form (26) may not exist. Nevertheless, it can be proved (see Lemma [ in Section VI) that the system (23) has a fundamental matrix

$$
\hat{Y}(x, \lambda)=G(\lambda) \sum_{n=0}^{\infty} H_{n}(\lambda)(1-x)^{n}(1-x)^{D}(1-x)^{J(\lambda)},
$$

in a complex neighbourhood of $x=1$, where $D:=\operatorname{diag}(-1, k-1), H_{0}(\lambda)=I$ and

$$
G(\lambda)=\left(\begin{array}{cc}
0 & \kappa+\frac{1}{2} \\
1 & \mu-\lambda
\end{array}\right), \quad J(\lambda)=\left(\begin{array}{cc}
0 & 0 \\
q(\lambda) & 0
\end{array}\right)
$$

with some $q(\lambda) \in \mathbb{C}$. In particular, we can write $\hat{Y}$ in the form

$$
\hat{Y}(x, \lambda)=\hat{H}(x, \lambda)(1-x)^{\tilde{J}(\lambda)}, \quad \hat{H}(x, \lambda)=\sum_{n=0}^{\infty}(1-x)^{n} D_{n}(\lambda),
$$

where

$$
D_{0}(\lambda)=\left(\begin{array}{ll}
0 & 0 \\
1 & 0
\end{array}\right), \quad \tilde{J}(\lambda)=\left(\begin{array}{cc}
-1 & 0 \\
q(\lambda) & -1
\end{array}\right)
$$

Since $\hat{y}$ solves the system (23), there exists a vector $c(\lambda) \in \mathbb{C}^{2}$ such that $\hat{y}(x, \lambda)=\hat{Y}(x, \lambda) c(\lambda)$, and [21, Theorem 1.1] implies

$$
d_{n}(\lambda)=D_{0}(\lambda) \frac{1}{\Gamma}(-\tilde{J}(\lambda)) \Gamma(n+1) \frac{1}{\Gamma}(n-\tilde{J}(\lambda)) c(\lambda)+\mathcal{O}\left(n^{\delta-1}\right)
$$

for arbitrary $\delta>0$. For the definition and discussion of the reciprocal Gamma function for matrices we refer to the Appendix in 21]. Particularly, for the Jordan type matrices $-\tilde{J}(\lambda)$ and $n-\tilde{J}(\lambda)$ i we obtain

$$
\frac{1}{\Gamma}(-\tilde{J}(\lambda))=\left(\begin{array}{ll}
1 & 0 \\
* & 1
\end{array}\right), \quad \frac{1}{\Gamma}(n-\tilde{J}(\lambda))=\left(\begin{array}{cc}
\frac{1}{\Gamma(n+1)} & 0 \\
* & \frac{1}{\Gamma(n+1)}
\end{array}\right) .
$$

Now, if $\gamma_{1}(\lambda)$ denotes the first component of $c(\lambda)$, then (28) implies (27). Since $\lambda$ is an eigenvalue of $A(\kappa ; \mu, \nu)$ if and only if $\gamma_{1}(\lambda)=0$, the proof of Lemma B is complete. 


\section{A PARTIAL DIFFERENTIAL EQUATION FOR THE EIGENVALUES}

Theorem 1 For fixed $\kappa \in\left[\frac{1}{2}, \infty\right)$ and $j \in \mathbb{Z} \backslash\{0\}$, the $j$-th eigenvalue $\lambda=\lambda_{j}(\kappa ; \mu, \nu)$ of $A$ is an analytical function in $(\mu, \nu) \in \mathbb{R}^{2}$ satisfying the first order quasi-linear partial differential equation

$$
(\mu-2 \nu \lambda) \frac{\partial \lambda}{\partial \mu}+(\nu-2 \mu \lambda) \frac{\partial \lambda}{\partial \nu}+2 \kappa \mu+2 \mu \nu=0,
$$

where $\lambda_{j}(\kappa ; 0,0)$ is given by (7).

Proof. Let

$$
S(\theta)=:\left(\begin{array}{c}
S_{1}(\theta) \\
S_{2}(\theta)
\end{array}\right), \quad \theta \in(0, \pi)
$$

be that eigenfunction of $A(\kappa ; \mu, \nu)$ for the eigenvalue $\lambda=\lambda_{j}(\kappa ; \mu, \nu)$ which is normalised by the condition $(S, S)=1$. Introducing the functions

$$
U(\theta):=S_{1}(\theta)^{2}+S_{2}(\theta)^{2}, \quad V(\theta):=S_{2}(\theta)^{2}-S_{1}(\theta)^{2}, \quad W(\theta):=2 S_{1}(\theta) S_{2}(\theta),
$$

a straightforward calculation shows that $U, V$, and $W$ are solutions of the system of differential equations

$$
\begin{aligned}
U^{\prime}(\theta) & =2\left(\nu \sin \theta+\frac{\kappa}{\sin \theta}\right) V(\theta)+2 \mu \cos \theta W(\theta), \\
V^{\prime}(\theta) & =2\left(\nu \sin \theta+\frac{\kappa}{\sin \theta}\right) U(\theta)+2 \lambda W(\theta), \\
W^{\prime}(\theta) & =2 \mu \cos \theta U(\theta)-2 \lambda V(\theta) .
\end{aligned}
$$

Now, from analytic perturbation theory (compare [14, Chap. VII, §3, Sec. 4]) it follows that

$$
\begin{aligned}
& \frac{\partial \lambda}{\partial \mu}=\left(\frac{\partial A}{\partial \mu} S, S\right)=\int_{0}^{\pi} S(\theta)^{*}\left(\begin{array}{cc}
-\cos \theta & 0 \\
0 & \cos \theta
\end{array}\right) S(\theta) \mathrm{d} \theta=\int_{0}^{\pi} \cos \theta V(\theta) \mathrm{d} \theta, \\
& \frac{\partial \lambda}{\partial \nu}=\left(\frac{\partial A}{\partial \nu} S, S\right)=\int_{0}^{\pi} S(\theta)^{*}\left(\begin{array}{cc}
0 & -\sin \theta \\
-\sin \theta & 0
\end{array}\right) S(\theta) \mathrm{d} \theta=-\int_{0}^{\pi} \sin \theta W(\theta) \mathrm{d} \theta .
\end{aligned}
$$

In addition, from (19) we obtain the estimates

$$
|U(\theta)|,|V(\theta)|,|W(\theta)| \leq C \sin ^{2 \kappa} \theta
$$

with some constant $C>0$. Since $\kappa$ is positive, $U, V$ and $W$ vanish at $\theta=0$ and $\theta=\pi$. If we integrate (33) by parts and replace $V^{\prime}(\theta)$ with the r.h.s. of (131), then we get

$$
\begin{aligned}
\frac{\partial \lambda}{\partial \mu} & =-\int_{0}^{\pi} \sin \theta V^{\prime}(\theta) \mathrm{d} \theta=-\int_{0}^{\pi}\left(2 \nu \sin ^{2} \theta+2 \kappa\right) U(\theta)+2 \lambda \sin \theta W(\theta) \mathrm{d} \theta \\
& =-(2 \nu+2 \kappa) \int_{0}^{\pi} U(\theta) \mathrm{d} \theta-2 \lambda \int_{0}^{\pi} \sin \theta W(\theta) \mathrm{d} \theta+2 \nu \int_{0}^{\pi} \cos ^{2} \theta U(\theta) \mathrm{d} \theta .
\end{aligned}
$$

Taking into account that

$$
\int_{0}^{\pi} U(\theta) \mathrm{d} \theta=(S, S)=1, \quad \int_{0}^{\pi} \sin \theta W(\theta) \mathrm{d} \theta=-\frac{\partial \lambda}{\partial \nu}
$$

we have

$$
\mu \frac{\partial \lambda}{\partial \mu}=-\mu(2 \nu+2 \kappa)+2 \mu \lambda \frac{\partial \lambda}{\partial \nu}+2 \mu \nu \int_{0}^{\pi} \cos ^{2} \theta U(\theta) \mathrm{d} \theta
$$

Moreover, equation (32) implies

$$
2 \mu \cos ^{2} \theta U(\theta)=\cos \theta W^{\prime}(\theta)+2 \lambda \cos \theta V(\theta)
$$


and integration by parts gives

$$
\begin{aligned}
2 \mu \nu \int_{0}^{\pi} \cos ^{2} \theta U(\theta) \mathrm{d} \theta & =\nu \int_{0}^{\pi} \cos \theta W^{\prime}(\theta) \mathrm{d} \theta+2 \nu \lambda \int_{0}^{\pi} \cos \theta V(\theta) \mathrm{d} \theta \\
& =\nu \int_{0}^{\pi} \sin \theta W(\theta) \mathrm{d} \theta+2 \nu \lambda \int_{0}^{\pi} \cos \theta V(\theta) \mathrm{d} \theta=-\nu \frac{\partial \lambda}{\partial \nu}+2 \nu \lambda \frac{\partial \lambda}{\partial \mu} .
\end{aligned}
$$

Replacing the last term on the r.h.s. of (35) with (36), we obtain exactly the partial differential equation (29).

The PDE (29) can be used in order to derive a power series expansion for $\lambda_{j}$ with respect to $\mu$ and $\nu$. For this purpose we introduce the new coordinates (compare [23])

$$
\alpha:=\nu-\mu, \quad \beta:=\nu+\mu .
$$

Then $\hat{\lambda}(\alpha, \beta):=\lambda_{j}\left(\kappa ; \frac{\beta-\alpha}{2}, \frac{\beta+\alpha}{2}\right)$ is a solution of the transformed partial differential equation

$$
\alpha(1+2 \hat{\lambda}) \frac{\partial \hat{\lambda}}{\partial \alpha}+\beta(1-2 \hat{\lambda}) \frac{\partial \hat{\lambda}}{\partial \beta}=\kappa(\alpha-\beta)+\frac{1}{2}\left(\alpha^{2}-\beta^{2}\right)
$$

where $\hat{\lambda}(0,0)=\lambda_{j}(\kappa ; 0,0)$ is given by (7). As $\hat{\lambda}$ depends analytically on $(\alpha, \beta)$, there exists a series expansion for $\hat{\lambda}$ of the form

$$
\hat{\lambda}(\alpha, \beta)=\sum_{m, n=0}^{\infty} c_{m, n} \alpha^{m} \beta^{n}
$$

(for clarity, the indices $\kappa$ and $j$ in the coefficients $c_{m, n}$ and in the function $\hat{\lambda}$ have been omitted). Furthermore, (37) is equivalent to

$$
\alpha\left(\frac{\partial \hat{\lambda}}{\partial \alpha}+\frac{\partial \hat{\lambda}^{2}}{\partial \alpha}\right)+\beta\left(\frac{\partial \hat{\lambda}}{\partial \beta}-\frac{\partial \hat{\lambda}^{2}}{\partial \beta}\right)=\kappa(\alpha-\beta)+\frac{1}{2}\left(\alpha^{2}-\beta^{2}\right)
$$

and since

$$
\hat{\lambda}(\alpha, \beta)^{2}=\sum_{m, n=0}^{\infty}\left(\sum_{r=0}^{m} \sum_{s=0}^{n} c_{r, s} c_{m-r, n-s}\right) \alpha^{m} \beta^{n}
$$

we obtain the identity

$$
\sum_{m, n=0}^{\infty}\left((m+n) c_{m, n}+(m-n) \sum_{r=0}^{m} \sum_{s=0}^{n} c_{r, s} c_{m-r, n-s}\right) \alpha^{m} \beta^{n}=\kappa(\alpha-\beta)+\frac{1}{2}\left(\alpha^{2}-\beta^{2}\right) .
$$

Comparing the terms of equal order in $\alpha$ and $\beta$, it follows that

$$
\begin{gathered}
c_{0,0}=\lambda_{j}(\kappa ; 0,0)=: c_{0}, \quad c_{1,0}=\frac{\kappa}{2 c_{0}+1}, \quad c_{0,1}=\frac{\kappa}{2 c_{0}-1}, \\
c_{2,0}=\frac{\left(2 c_{0}+1\right)^{2}-4 \kappa^{2}}{4\left(2 c_{0}+1\right)^{3}}, \quad c_{1,1}=0, \quad c_{0,2}=\frac{\left(2 c_{0}-1\right)^{2}-4 \kappa^{2}}{4\left(2 c_{0}-1\right)^{3}},
\end{gathered}
$$

and for $m+n>2$ the coefficients $c_{m, n}$ satisfy

$$
\left((m+n)+2 c_{0}(m-n)\right) c_{m, n}=(n-m) \sum_{(r, s) \in \llbracket m, n \rrbracket} c_{r, s} c_{m-r, n-s}
$$

where $\llbracket m, n \rrbracket$ denotes the set of all pairs $(r, s) \in \mathbb{Z}^{2}$ with $0 \leq r \leq m, 0 \leq s \leq n$ and $0<r+s<m+n$. In particular, if $m=n>0$, then we get $2 n c_{n, n}=0$, which implies

$$
c_{n, n}=0 \quad \text { for all } n>0
$$


Moreover, if $\kappa$ is not a rational number, i.e., $\kappa \in\left[\frac{1}{2}, \infty\right) \backslash \mathbb{Q}$, then the initial value $c_{0}$ is not a rational number, and we have $(m+n)+2 c_{0}(m-n) \neq 0$ for all $(m, n) \in \mathbb{Z}^{2}$ with $m+n>2$. In this case (40) gives a recurrence formula for all coefficients of the power series expansion (38).

Now, suppose that $\kappa \in\left[\frac{1}{2}, \infty\right) \cap \mathbb{Q}$. Then $c_{0}$ is a rational number with $\left|c_{0}\right| \geq 1$, and we get $\frac{2 c_{0}-1}{2 c_{0}+1}=\frac{p}{q}$ with some coprime integers $p$ and $q$. Now, the prefactor on the l.h.s. of (40) becomes zero if and only if $m=\ell p, n=\ell q$ with some positive integer $\ell$, and thus the coefficients $c_{\ell p, \ell q}$ are not determined by (40). However, we can by-pass this problem if we regard $\kappa$ as an additional parameter in our eigenvalue problem. Since the coefficient matrix of (11) depends holomorphically on $\kappa \in \mathbb{C}^{+}:=\{z \in \mathbb{C}: \operatorname{Re} z>0\}$ and $(\lambda, \mu, \nu) \in \mathbb{C}^{3}$, we obtain in a similar way as described in Section 1 that $h$ in (14) and therefore $\Delta=\Delta(\kappa ; \lambda ; \mu, \nu)$ given by (17) is a holomorphic function on $\mathbb{C}^{+} \times \mathbb{C}^{3}$. Moreover, in the same way as in the proof of Lemma 1] we can show that for fixed $\kappa \in\left[\frac{1}{2}, \infty\right)$ and $(\mu, \nu) \in \mathbb{C}^{2}$ the eigenvalues of $A(\kappa ; \mu, \nu)$ coincide with the zeros of the function $\lambda \longmapsto \Delta(\kappa ; \lambda ; \mu, \nu)$. In the case $(\kappa, \mu, \nu) \in\left[\frac{1}{2}, \infty\right) \times \mathbb{R}^{2}$ these zeros are simple, since $A(\kappa ; \mu, \nu)$ has only simple eigenvalues. Hence, by solving the equation $\Delta(\kappa ; \lambda ; \mu, \nu)=0$, we find that an eigenvalue $\lambda_{j}(\kappa ; \mu, \nu)$ is a holomorphic function in a complex neighbourhood of $\left[\frac{1}{2}, \infty\right) \times \mathbb{R}^{2}$. In particular, $\hat{\lambda}$ depends holomorphically on $(\kappa ; \alpha, \beta)$, and for a given $\kappa \in\left[\frac{1}{2}, \infty\right)$, there exists a power series expansion of the form

$$
\hat{\lambda}(\kappa+\varepsilon ; \alpha, \beta)=\sum_{l, m, n=0}^{\infty} c_{m, n}^{(l)} \varepsilon^{l} \alpha^{m} \beta^{n}
$$

in a neighbourhood of $(\kappa, 0,0)$. In the following we derive a recurrence relation for the coefficients $c_{m, n}^{(l)}$. Since

$$
\hat{\lambda}(\kappa+\varepsilon ; \alpha, \beta)^{2}=\sum_{l, m, n=0}^{\infty}\left(\sum_{t=0}^{l} \sum_{r=0}^{m} \sum_{s=0}^{n} c_{r, s}^{(t)} c_{m-r, n-s}^{(l-t)}\right) \varepsilon^{l} \alpha^{m} \beta^{n},
$$

from (39) it follows that

$$
\sum_{l, m, n=0}^{\infty}\left((m+n) c_{m, n}^{(l)}+(m-n) \sum_{t=0}^{l} \sum_{r=0}^{m} \sum_{s=0}^{n} c_{r, s}^{(t)} c_{m-r, n-s}^{(l-t)}\right) \varepsilon^{l} \alpha^{m} \beta^{n}=\kappa(\alpha-\beta)+\varepsilon(\alpha-\beta)+\frac{1}{2}\left(\alpha^{2}-\beta^{2}\right) .
$$

Moreover, (17) implies that

$$
c_{0,0}^{(l)}=\frac{1}{l !} \frac{\partial^{l} \lambda}{\partial \kappa^{l}}(\kappa ; 0,0)=\left\{\begin{aligned}
\operatorname{sgn}(j)\left(\kappa-\frac{1}{2}+|j|\right), & \text { if } l=0 \\
\operatorname{sgn}(j), & \text { if } l=1 \\
0, & \text { if } l>1 .
\end{aligned}\right.
$$

Comparing the terms of equal order in (42), we obtain

$$
\begin{gathered}
c_{0,0}^{(0)}=\lambda_{j}(\kappa ; 0,0)=: c_{0}, \quad c_{1,0}^{(0)}=\frac{\kappa}{2 c_{0}+1}, \quad c_{0,1}^{(0)}=\frac{\kappa}{2 c_{0}-1}, \\
c_{2,0}^{(0)}=\frac{\left(2 c_{0}+1\right)^{2}-4 \kappa^{2}}{4\left(2 c_{0}+1\right)^{3}}, \quad c_{1,1}^{(0)}=0, \quad c_{0,2}^{(0)}=\frac{\left(2 c_{0}-1\right)^{2}-4 \kappa^{2}}{4\left(2 c_{0}-1\right)^{3}} \\
c_{1,0}^{(1)}=\frac{2 c_{0}+1-2 \operatorname{sgn}(j) \kappa}{\left(2 c_{0}+1\right)^{2}}, \quad c_{0,1}^{(1)}=\frac{2 c_{0}-1-2 \operatorname{sgn}(j) \kappa}{\left(2 c_{0}-1\right)^{2}},
\end{gathered}
$$

while the remaining coefficients are determined by the identity

$$
\left((m+n)+2 c_{0}(m-n)\right) c_{m, n}^{(l)}+(m-n) \sum_{(t, r, s) \in \llbracket l, m, n \rrbracket} c_{r, s}^{(t)} c_{m-r, n-s}^{(l-t)}=0, \quad l+m+n>2 .
$$

Here $\llbracket l, m, n \rrbracket$ denotes the set of all triples $(t, r, s) \in \mathbb{Z}^{3}$ with $0 \leq t \leq l, 0 \leq r \leq m, 0 \leq s \leq n$, and $0<t+r+s<$ $l+m+n$. In the case $(m+n)+2 c_{0}(m-n)=0$, the prefactor of $c_{m, n}^{(l)}$ in (43) vanishes, and since $m-n \neq 0$, we get for $l>0$

$$
0=\sum_{(t, r, s) \in \llbracket l, m, n \rrbracket} c_{r, s}^{(t)} c_{m-r, n-s}^{(l-t)}=2 c_{0,0}^{(1)} c_{m, n}^{(l-1)}+\sum_{(t, r, s) \in \llbracket l, m, n \rrbracket^{*}} c_{r, s}^{(t)} c_{m-r, n-s}^{(l-t)}
$$


where $\llbracket l, m, n \rrbracket^{*}:=\llbracket l, m, n \rrbracket \backslash\{(1,0,0),(l-1, m, n)\}$. Now, for all coefficients $c_{m, n}^{(l)}$ with $l+m+n>2$, (43) implies

$$
c_{m, n}^{(l)}=\frac{n-m}{(m+n)+2 c_{0}(m-n)} \sum_{(t, r, s) \in \llbracket l, m, n \rrbracket} c_{r, s}^{(t)} c_{m-r, n-s}^{(l-t)} \quad \text { if } \quad(m+n)+2 c_{0}(m-n) \neq 0,
$$

whereas (44) and $c_{0,0}^{(1)}=\operatorname{sgn}(j)$ yield

$$
c_{m, n}^{(l-1)}=-\frac{\operatorname{sgn}(j)}{2} \sum_{(t, r, s) \in \llbracket l, m, n \rrbracket^{*}} c_{r, s}^{(t)} c_{m-r, n-s}^{(l-t)} \quad \text { if } \quad(m+n)+2 c_{0}(m-n)=0 \text { and } l>1 .
$$

These recurrence relations can be used to determine all the coefficients $c_{m, n}=c_{m, n}^{(0)}$ of the power series expansion (38) in the case that $\kappa$ is a rational number.

Remark 1 A series expansion for the eigenvalues $\hat{\lambda}$ with respect to $(\alpha, \beta)$ has been given by Suffern, Fackerell $\mathcal{G}$ Cosgrove [23, Sec. 8], however, only the coefficients $c_{m, n}$ with $m+n \leq 5$ have been determined. Furthermore, Kalnins 8 Miller [1]] studied a series expansion $\lambda=\sum_{n=0}^{\infty} \lambda_{n} a^{n}$ for the eigenvalues in terms of the Kerr parameter a, but also in this paper only a finite number of coefficients $\lambda_{0}, \ldots, \lambda_{3}$ have been explicitly computed. A general recurrence relation for the coefficients of (38) could not be found in the literature. Moreover, the problem of dividing by numbers which may be zero has not been noticed in [23] and [13]. Finally, it should be noted that some of the diagonal entries $c_{n, n}$ for $n>0$ in [2], Table I] are not equal to zero, in contrast to our result (41).

\section{SOLUTION OF THE PDE BY THE METHOD OF CHARACTERISTICS}

In this section we study the PDE (29) for real parameters $(\mu, \nu) \in \mathbb{R}^{2}$ and fixed $\kappa \in\left[\frac{1}{2}, \infty\right)$ by the method of characteristics. In particular, we obtain an exact formula for the eigenvalues in the case $|\mu|=|\nu|$, and for $|\mu| \neq|\nu|$, it turns out that the characteristic equations can be reduced to the third Painlevé equation.

Theorem 2 Let $\kappa \in\left[\frac{1}{2}, \infty\right), j \in \mathbb{Z} \backslash\{0\}$ and $\tau \in\{-1,+1\}$ be fixed. Then

$$
\lambda_{j}(\kappa ; \mu, \tau \mu)=\frac{\tau}{2}+\operatorname{sgn}(j) \sqrt{\left(\lambda_{j}(\kappa ; 0,0)-\frac{\tau}{2}\right)^{2}+2 \tau \kappa \mu+\mu^{2}},
$$

where $\lambda_{j}(\kappa ; 0,0)=\operatorname{sgn}(j)\left(\kappa-\frac{1}{2}+|j|\right)$. In particular, if $j=\tau$, then

$$
\lambda_{j}(\kappa ; \mu, \tau \mu)=\tau\left(\kappa+\frac{1}{2}\right)+\mu .
$$

Proof. According to Theorem [1 the function $\lambda(\mu, \nu):=\lambda_{j}(\kappa ; \mu, \nu)$ solves the partial differential equation (29). Defining $w(\mu):=\lambda(\mu, \tau \mu), \mu \in \mathbb{R}$, for some fixed $\tau \in\{-1,+1\}$, we obtain

$$
w^{\prime}(\mu)=\frac{\partial \lambda}{\partial \mu}(\mu, \tau \mu)+\tau \frac{\partial \lambda}{\partial \nu}(\mu, \tau \mu),
$$

and with the help of (29) it can be shown that

$$
\mu w^{\prime}(\mu)-2 \tau \mu w(\mu) w^{\prime}(\mu)=-2 \kappa \mu-2 \tau \mu^{2} .
$$

Dividing the above differential equation by $-\tau \mu$ and integrating gives

$$
\left(w(\mu)-\frac{\tau}{2}\right)^{2}=C+2 \tau \kappa \mu+\mu^{2}, \quad \mu \in \mathbb{R},
$$

where the constant of integration $C$ is uniquely determined by

$$
C=\left(w(0)-\frac{\tau}{2}\right)^{2}=\left(\lambda_{j}(\kappa ; 0,0)-\frac{\tau}{2}\right)^{2} .
$$

Now, from (46) it follows that

$$
w(\mu)=\frac{\tau}{2}+\varepsilon \sqrt{\left(\lambda_{j}(\kappa ; 0,0)-\frac{\tau}{2}\right)^{2}+2 \tau \kappa \mu+\mu^{2}}
$$


with some $\varepsilon \in\{-1,+1\}$ and the square root assumed to be non-negative. We have to take the sign $\varepsilon$ such that the l.h.s. of (47) is analytic and coincides with $\lambda_{j}(\kappa ; 0,0)$ at the point $\mu=0$. If $j=\tau$, then $\lambda_{j}(\kappa ; 0,0)=\tau\left(\kappa+\frac{1}{2}\right)$, and (47) implies $w(\mu)=\frac{\tau}{2}+\varepsilon(\tau \kappa+\mu)$. Inserting $\mu=0$, it follows that $\varepsilon=\tau$, i.e., $w(\mu)=\tau\left(\kappa+\frac{1}{2}\right)+\mu$. In the case $j \neq \tau$ we have $\left|\lambda_{j}(\kappa ; 0,0)-\frac{\tau}{2}\right| \geq \kappa+1$ and thus the radicand in (47) is positive for all $\mu \in \mathbb{R}$. Moreover, by means of

$$
\lambda_{j}(\kappa ; 0,0)=w(0)=\frac{\tau}{2}+\varepsilon \sqrt{\left(\lambda_{j}(\kappa ; 0,0)-\frac{\tau}{2}\right)^{2}}=\frac{\tau}{2}+\varepsilon\left|\lambda_{j}(\kappa ; 0,0)-\frac{\tau}{2}\right|
$$

and (77), we get $\varepsilon=\operatorname{sgn}\left(\lambda_{j}(\kappa ; 0,0)-\frac{\tau}{2}\right)=\operatorname{sgn}(j)$, which completes the proof.

Remark 2 For a given half-integer $\kappa$ and $\mu=\nu$, this result has been shown by Suffern, Fackerell \& Cosgrove using a power series expansion for the eigenfunctions of (11) - (2) in terms of hypergeometric functions (see [25, Sec. 3-5]). Here, we obtained the formula for $\lambda_{j}(\kappa ; \mu, \pm \mu)$ as an immediate consequence of the partial differential equation (29). Moreover, it should be noted that the formula [1, (54)] given by Chakrabarti is not correct.

Now, let us consider the case $|\mu| \neq|\nu|$. For this reason, we introduce new coordinates $(t, v) \in(0, \infty) \times(\mathbb{R} \backslash\{0\})$ by

$$
\mu(t, v)=\frac{t}{2}\left(v+\frac{\sigma}{v}\right), \quad \nu(t, v)=\frac{t}{2}\left(v-\frac{\sigma}{v}\right)
$$

with some fixed $\sigma \in\{-1,+1\}$ (note that $\sigma= \pm 1$ corresponds to the cases $|\mu|>|\nu|$ and $|\mu|<|\nu|$, respectively; moreover, this transformation maps $v=$ const. onto lines in the $(\mu, \nu)$-plane starting at the origin, while the curves $t=$ const. are mapped onto hyperboles). By setting $w(t, v)=\lambda(\mu, \nu)$, we have

$$
\begin{aligned}
& \frac{\partial w}{\partial t}=\frac{1}{2}\left(v+\frac{\sigma}{v}\right) \frac{\partial \lambda}{\partial \mu}+\frac{1}{2}\left(v-\frac{\sigma}{v}\right) \frac{\partial \lambda}{\partial \nu}=\frac{1}{t}\left(\mu \frac{\partial \lambda}{\partial \mu}+\nu \frac{\partial \lambda}{\partial \nu}\right), \\
& \frac{\partial w}{\partial v}=\frac{t}{2}\left(1-\frac{\sigma}{v^{2}}\right) \frac{\partial \lambda}{\partial \mu}+\frac{t}{2}\left(1+\frac{\sigma}{v^{2}}\right) \frac{\partial \lambda}{\partial \nu}=\frac{1}{v}\left(\nu \frac{\partial \lambda}{\partial \mu}+\mu \frac{\partial \lambda}{\partial \nu}\right),
\end{aligned}
$$

and (29) becomes

$$
\frac{\partial w}{\partial t}-\frac{2 v w}{t} \frac{\partial w}{\partial v}+\kappa\left(v+\frac{\sigma}{v}\right)+\frac{t}{2}\left(v^{2}-\frac{1}{v^{2}}\right)=0 .
$$

The characteristic equations of this PDE are given by

$$
\begin{aligned}
v^{\prime}(t) & =-\frac{2 v(t) w(t)}{t}, \\
w^{\prime}(t) & =-\kappa\left(v(t)+\frac{\sigma}{v(t)}\right)-\frac{t}{2}\left(v(t)^{2}-\frac{1}{v(t)^{2}}\right) .
\end{aligned}
$$

From (50) we obtain that $w(t)=-\frac{t v^{\prime}(t)}{2 v(t)}$, and (151) implies

$$
\frac{v^{\prime}(t)}{2 v(t)}+\frac{t v^{\prime \prime}(t)}{2 v(t)}-\frac{t v^{\prime}(t)^{2}}{2 v(t)^{2}}=\kappa\left(v(t)+\frac{\sigma}{v(t)}\right)+\frac{t}{2}\left(v(t)^{2}-\frac{1}{v(t)^{2}}\right) .
$$

Multiplying the above differential equation with $2 v(t)^{2}$, we get the following third Painlevé equation

$$
t v v^{\prime \prime}-t\left(v^{\prime}\right)^{2}+v v^{\prime}-2 \kappa\left(v^{2}+\sigma\right) v-t\left(v^{4}-1\right)=0,
$$

with parameters $\alpha=\sigma \beta=2 \kappa$ and $\gamma=-\delta=1$ (see, for example, [18] or [16]). For further details on the Painlevé III we refer to e.g. [4], [26] and [8].

In general, Painlevé III is not solvable in terms of elementary functions, and therefore we cannot expect a closed expression for the eigenvalues of $A(\kappa ; \mu, \nu)$ in the case $|\mu| \neq|\nu|$. On the other hand, for particular values of $\kappa$ there exist so-called special integrals of polynomial type for this equation, i.e., polynomials $Q$ in $t, v$ and $v^{\prime}$ with the property that every solution of the differential equation $Q\left(t, v, v^{\prime}\right)=0$ satisfies (52). As it will be shown below, such special integrals are related to algebraic solutions of the PDE (29), i.e., solutions, which are zeros of a polynomial in $\lambda$ with rational coefficients in $\mu$ and $\nu$. Moreover, taking into account that the eigenvalues $\lambda_{j}(\kappa ; \mu, \tau \mu), \tau \in\{-1,1\}$, of $A(\kappa ; \mu, \tau \mu)$ satisfy the quadratic equation

$$
\left(\lambda-\frac{\tau}{2}\right)^{2}=C+2 \tau \kappa \mu+\mu^{2} \quad \text { with } \quad C:=\left(\lambda_{j}(\kappa ; 0,0)-\frac{\tau}{2}\right)^{2},
$$

the question arises if such an algebraic expression for the eigenvalues of $A(\kappa ; \mu, \nu)$ exists in the case $|\mu| \neq|\nu|$. A first step towards the answer of this problem is given by the next Lemma. 
Lemma 4 Suppose that there exists a polynomial

$$
P(\lambda ; \mu, \nu)=\sum_{n=0}^{N} P_{n}(\mu, \nu) \lambda^{n}, \quad P_{N} \equiv 1,
$$

of degree $N>0$ in $\lambda$ with rational coefficients $P_{n}$ in $\mu$ and $\nu$ such that the zeros $z_{j}(\mu, \nu), j=1, \ldots N$, of $P(\cdot ; \mu, \nu)$ are simple, and that the functions $\lambda=z_{j}$ are solutions of the partial differential equation (29). Then $\kappa$ is a half-integer. Moreover, if $N=1$ or $N=2$, then $\kappa=\frac{1}{2}$ and $P(\lambda ; \mu, \nu)=(\lambda+\mu)^{N}$.

Proof. Let $Q\left(t, v, v^{\prime}\right)=\sum_{n=0}^{N} Q_{n}(t, v)\left(v^{\prime}\right)^{n}$ be the polynomial in $v^{\prime}$ with coefficients

$$
Q_{n}(t, v):=\left(-\frac{2 v}{t}\right)^{N-n} P_{n}(\mu(t, v), \nu(t, v)), \quad n=0, \ldots, N,
$$

where $\mu(t, v)$ and $\nu(t, v)$ are given by (48). Note that the $Q_{n}$ are again rational functions in $t$ and $v$. Moreover, let $v: \mathfrak{D} \longrightarrow \mathbb{R} \backslash\{0\}$ be any solution of the first order ODE $Q\left(t, v, v^{\prime}\right)=0$ on some interval $\mathfrak{D} \subset \mathbb{R} \backslash\{0\}$. For the function

$$
w(t)=-\frac{t v^{\prime}(t)}{2 v(t)}, \quad t \in \mathfrak{D},
$$

we obtain

$$
0=Q\left(t, v(t), v^{\prime}(t)\right)=\sum_{n=0}^{N} Q_{n}(t, v(t))\left(-\frac{2 v(t) w(t)}{t}\right)^{n}=\left(-\frac{2 v(t)}{t}\right)^{N} P(w(t) ; \mu(t, v(t)), \nu(t, v(t))),
$$

and thus $w(t)$ is a zero of $P(\cdot ; \mu(t, v(t)), \nu(t, v(t)))$ for each $t \in \mathfrak{D}$. Since the zeros of this polynomial depend analytically on the parameter $t$ according to the implicit function theorem, there exists an index $j \in\{1, \ldots, N\}$ such that $w(t)=z_{j}(\mu(t, v(t)), \nu(t, v(t)))$ for all $t \in \mathfrak{D}$. Furthermore, as $z_{j}$ solves the PDE (29), it follows that $(t, v(t), w(t)), t \in \mathfrak{D}$, is a characteristic curve of (49), and thus $v$ is a solution of (52). Hence, $Q\left(t, v, v^{\prime}\right)=0$ implies (52), and therefore $Q$ is a special integral of rational type for this Painlevé III. Multiplying $Q\left(t, v, v^{\prime}\right)$ by an appropriate polynomial $r(t, v)$ in $t$ and $v$, we obtain that $\tilde{Q}\left(t, v, v^{\prime}\right):=r(t, v) Q\left(t, v, v^{\prime}\right)$ is a special integral of polynomial type of degree $N$ with respect to $v^{\prime}$. Now, 16, Theorem 2] yields that such a special integral exists if and only if $2 \kappa \pm 2 \sigma \kappa=2(2 k-1)$ with some integer $k$, i.e., $\kappa=k-\frac{1}{2}$ is a half-integer. In addition, by [16, Lemma 3], the relation $(\sigma q-p) \kappa=N$ has to be satisfied for some integers $p, q \in\{-N,-N+2, \ldots, N-2, N\}$. In the case $N=1$ or $N=2$, these conditions imply $\kappa=\frac{1}{2}$, and the corresponding special integrals of polynomial type are explicitly known, namely $r(t) v^{s}\left(v^{\prime}+v^{2}+\sigma\right)^{N}$, where $r$ is some polynomial in $t$, and $s$ is an integer (compare [16, Section 2]). Hence, $Q\left(t, v, v^{\prime}\right)=\left(v^{\prime}+v^{2}+\sigma\right)^{N}$ and

$$
P(w(t) ; \mu(t, v(t)), \nu(t, v(t)))=\left(-\frac{t}{2 v(t)}\right)^{N} Q(t, v(t))=(w(t)+\mu((t, v(t))))^{N}, \quad t \in \mathfrak{D},
$$

which yields $P(\lambda ; \mu, \nu)=(\lambda+\mu)^{N}$ if $N=1$ or $N=2$.

As a consequence of this Lemma, if a solution $\lambda(\mu, \nu)$ of the PDE (29) is a zero of a linear or quadratic polynomial with rational coefficients in $\mu$ and $\nu$, then $\kappa=\frac{1}{2}$ and $\lambda(\mu, \nu)=-\mu$. In fact, the function $\lambda(\mu, \nu)=-\mu$ solves (29) for $\kappa=\frac{1}{2}$, but since $\lambda(0,0)=0$ and the spectrum of $A\left(\frac{1}{2} ; 0,0\right)$ is given by $\mathbb{Z} \backslash\{0\}$, it is not an eigenvalue of $A\left(\frac{1}{2} ; \mu, \nu\right)$ for any $(\mu, \nu) \in \mathbb{R}^{2}$. The following considerations show that this solution is nevertheless of interest.

\section{MONODROMY EIGENVALUES}

In this section we consider the case that $\kappa$ is a positive half-integer, i.e., $\kappa=k-\frac{1}{2}$ with some positive integer $k$, and we assume that the matrix $C$ defined in (12) has distinct eigenvalues, i.e., $\mu^{2} \neq \nu^{2}$. For such $\kappa$ and $(\mu, \nu)$ there is in addition to the classical eigenvalues of $A(\kappa ; \mu, \nu)$ another type of "special values" which we call monodromy eigenvalues. In order to introduce this concept, we first recall the characterisation of eigenvalues according to Lemma 1 A point $\lambda$ is an eigenvalue of $A(\kappa ; \mu, \nu)$ if and only if the system (11) has a nontrivial solution of the form

$$
y(x)=[x(1-x)]^{\frac{\kappa}{2}+\frac{1}{4}} \eta(x)
$$


where $\eta: \mathbb{C} \longrightarrow \mathbb{C}^{2}$ is an entire vector function. Now, as the difference of the characteristic values $\pm\left(\frac{\kappa}{2}+\frac{1}{4}\right)$ at 0 and 1 is an integer, the differential equation (11) has a fundamental matrix of the form

$$
Y(x)=[x(1-x)]^{-\frac{\kappa}{2}-\frac{1}{4}} H(x)
$$

where $H(x)=H_{a}(x)(x-a)^{J_{a}}$ in $\mathfrak{B}_{a}, a \in\{0,1\}$, with some holomorphic function $H_{a}: \mathfrak{B}_{a} \longrightarrow \mathrm{M}_{2}(\mathbb{C})$ and a Jordan matrix $J_{a}$ (see [24, Theorem 5.6]). Hence, the matrix function $H$ is in general not holomorphic in $\mathfrak{B}_{0} \cup \mathfrak{B}_{1}$ since it involves logarithmic terms. In the following, a point $\lambda \in \mathbb{C}$ is called monodromy eigenvalue of $A(\kappa ; \mu, \nu)$ if and only if the system (11) has a fundamental matrix of the form (55) with the property that $H: \mathbb{C} \longrightarrow \mathrm{M}_{2}(\mathbb{C})$ is an entire matrix function. Monodromy eigenvalues are characterised by the following Lemma.

Lemma 5 For a given half-integer $\kappa>0$ and $(\mu, \nu) \in \mathbb{C}^{2}$ with $\mu^{2} \neq \nu^{2}$, a point $\lambda \in \mathbb{C}$ is a monodromy eigenvalue of $A(\kappa ; \mu, \nu)$ if and only if the system (11) has a nontrivial solution of the form

$$
[x(1-x)]^{-\frac{\kappa}{2}-\frac{1}{4}} p(x) e^{2 t x}
$$

where $p: \mathbb{C} \longrightarrow \mathbb{C}^{2}$ is a polynomial vector function and $t= \pm \sqrt{\nu^{2}-\mu^{2}}$.

Proof. By means of the transformation $y(x)=x^{\alpha}(1-x)^{\alpha} \tilde{y}(x)$ with $\alpha:=\frac{\kappa}{2}+\frac{1}{4}$, the differential equation (11) is equivalent to the system

$$
\tilde{y}^{\prime}(x)=\left[\frac{1}{x} \tilde{B}_{0}+\frac{1}{x-1} \tilde{B}_{1}+C\right] \tilde{y}(x)
$$

where

$$
\tilde{B}_{0}=\left(\begin{array}{cc}
0 & \mu-\lambda \\
0 & k
\end{array}\right), \quad \tilde{B}_{1}=\left(\begin{array}{cc}
k & 0 \\
\mu-\lambda & 0
\end{array}\right) .
$$

Now, if $\lambda$ is a monodromy eigenvalue of $A(\kappa ; \mu, \nu)$, then the system (57) has a holomorphic fundamental matrix $H: \mathbb{C} \longrightarrow \mathrm{M}_{2}(\mathbb{C})$. Since the coefficient matrix of (57) is a rational matrix function which is bounded at infinity, an extension of Halphen's Theorem (see 6, Theorem 2.4]) implies that the system (57) has a fundamental matrix of the form $R(x) e^{D x}$ with some rational matrix function $R$ and $D:=\operatorname{diag}(-2 t, 2 t)$ (note that $\pm 2 t$ are the eigenvalues of $C$ ). Moreover, $R(x) e^{D x}=H(x) Q$ with some invertible matrix $Q$, and therefore $R(x)=H(x) Q e^{-D x}$ is an entire matrix function in $\mathbb{C}$. This implies that $R: \mathbb{C} \longrightarrow \mathrm{M}_{2}(\mathbb{C})$ is a polynomial. Vice versa, suppose that the system (11) has a nontrivial solution $y(x)=[x(1-x)]^{-\alpha} p(x) e^{2 t x}$ with some polynomial vector function $p: \mathbb{C} \longrightarrow \mathbb{C}^{2}$. Defining

$$
\tilde{y}(x):=e^{-2 t} K y(1-x)=[x(1-x)]^{-\alpha} K p(1-x) e^{-2 t x}
$$

with $K$ given by (20), it follows that $\tilde{y}$ is a solution of (11) which is linearly independent of $y$. Therefore, (11) has a fundamental matrix of the type (55), where $H(x)=\left(p(x) e^{2 t x}, K p(1-x) e^{-2 t x}\right)$ is an entire matrix function.

Theorem 3 For fixed $\kappa=k-\frac{1}{2}$ with a positive integer $k$ there exists a polynomial $P(\kappa ; \lambda ; \mu, \nu)$ of degree $2 k-1$ in $\lambda$ with polynomial coefficients in $\mu$ and $\nu$ such that for each $(\mu, \nu) \in \mathbb{C}^{2}$ with $\mu^{2} \neq \nu^{2}$ a point $\lambda \in \mathbb{C}$ is a monodromy eigenvalue of $A(\kappa ; \mu, \nu)$ if and only if $\lambda$ is a zero of $P(\kappa ; \cdot ; \mu, \nu)$. Moreover, the integers $1-k, \ldots, k-1$ are the zeros of $P(\kappa ; \cdot ; 0,0)$, and for $\kappa=\frac{1}{2}$ we obtain $P\left(\frac{1}{2} ; \lambda ; \mu, \nu\right)=\lambda+\mu$.

Proof. A point $\lambda$ is a monodromy eigenvalue of $A(\kappa ; \mu, \nu)$ if and only if the differential equation (57) has a nontrivial solution $p(x) e^{2 t x}$, where $p(x)=\sum_{n=0}^{N} p_{n} x^{n}, p_{N} \neq 0$, is a polynomial vector function, and $t= \pm \sqrt{\nu^{2}-\mu^{2}}$. In the following we assume $t=\sqrt{\nu^{2}-\mu^{2}}$ (the main branch of the square root) but all considerations remain valid if we replace $t$ with $-t$. If we set $\Lambda:=\lambda-\mu$ and $\tilde{C}:=C-t$, then the polynomial $p$ satisfies the differential equation

$$
p^{\prime}(x)=\left[\frac{1}{x} \tilde{B}_{0}+\frac{1}{x-1} \tilde{B}_{1}+\tilde{C}\right] p(x),
$$

where the coefficient matrices take the form

$$
\tilde{B}_{0}=\left(\begin{array}{cc}
0 & -\Lambda \\
0 & k
\end{array}\right), \quad \tilde{B}_{1}=\left(\begin{array}{cc}
k & 0 \\
-\Lambda & 0
\end{array}\right), \quad \tilde{C}=\left(\begin{array}{cc}
-2 \nu-2 t & -2 \mu \\
2 \mu & 2 \nu-2 t
\end{array}\right) .
$$


It is easy to see that the coefficients $p_{n} \in \mathbb{C}^{2}, n=0, \ldots, N$, form a nontrivial solution of the linear system of equations

$$
\begin{gathered}
\tilde{B}_{0} p_{0}=0, \quad\left(\tilde{B}_{0}-1\right) p_{1}+\tilde{S} p_{0}=0, \\
\left(\tilde{B}_{0}-n\right) p_{n}+(\tilde{S}+n-1) p_{n-1}-\tilde{C} p_{n-2}=0 \quad(n=2, \ldots, N), \\
(\tilde{S}+N) p_{N}-\tilde{C} p_{N-1}=0, \quad-\tilde{C} p_{N}=0,
\end{gathered}
$$

where

$$
\tilde{S}:=\tilde{C}-\tilde{B}_{0}-\tilde{B}_{1}=\left(\begin{array}{cc}
-2 \nu-2 t-k & -2 \mu+\Lambda \\
2 \mu+\Lambda & 2 \nu-2 t-k
\end{array}\right) .
$$

Multiplying the first equation in (62) from the left with the matrix $\tilde{C}+4 t$ and observing that $(\tilde{C}+4 t) \tilde{C}=0$, we get

$$
0=(\tilde{C}+4 t)(\tilde{S}+N) p_{N}=\left(\begin{array}{cc}
N-k & -\Lambda \\
-\Lambda & N-k
\end{array}\right) \tilde{C} p_{N}+4 t(N-k) p_{N}=4 t(N-k) p_{N} .
$$

Since $t \neq 0$ and $p_{N} \neq 0$, it follows that $N=k$. Due to technical reasons we have to distinguish between the cases $k \geq 2$ and $k=1$. We will proceed at first with a detailed proof for the more complicated case $k \geq 2$. Adding the second equation in (62) to the first one and then both equations in (62) to (61) for $n=N$, we obtain

$$
\begin{gathered}
\tilde{B}_{0} p_{0}=0, \quad\left(\tilde{B}_{0}-1\right) p_{1}+\tilde{S} p_{0}=0, \\
\left(\tilde{B}_{0}-n\right) p_{n}+(\tilde{S}+n-1) p_{n-1}-\tilde{C} p_{n-2}=0 \quad(n=2, \ldots, k-1), \\
-\tilde{B}_{1} p_{k}+\left(\begin{array}{cc}
-1 & \Lambda \\
\Lambda & -1
\end{array}\right) p_{k-1}-\tilde{C} p_{k-2}=0 \\
\left(\begin{array}{cc}
0 & \Lambda \\
\Lambda & 0
\end{array}\right) p_{k}-\tilde{C} p_{k-1}=0, \quad-\tilde{C} p_{k}=0 .
\end{gathered}
$$

The system above consists of $2 k+6$ linear equations for $2 k+2$ unknowns. In the following we prove that only $2 k+2$ of these equations are linearly independent. Summation of all equations (60) - 62) yields $-\tilde{B}_{1} \sum_{n=0}^{k} p_{n}=0$. Because of $\operatorname{rank}\left(\tilde{B}_{1}\right)=1$, it is possible to eliminate the second line of the first equation in 65 means of line transformations, and since also $\operatorname{rank}\left(\tilde{B}_{0}\right)=1$, we can delete the first line of the first equation in (63). Thus, the system (63) - (66) consists of at most $2 k+4$ linearly independent equations. In order to reduce the equations (66) further, we have to consider the cases $\nu-t \neq 0$ and $\nu-t=0$ separately. First, let us assume that $\nu-t \neq 0$. Multiplying the equations in (66) from the left by the invertible matrix

$$
T:=\left(\begin{array}{cc}
\nu-t & \mu \\
0 & 1
\end{array}\right)
$$

it follows that (66) is equivalent to

$$
\left(\begin{array}{ll}
0 & 0 \\
\Lambda & 0
\end{array}\right) p_{k}-\left(\begin{array}{cc}
0 & 0 \\
2 \mu & 2 \nu-2 t
\end{array}\right) p_{k-1}=0, \quad\left(\begin{array}{cc}
0 & 0 \\
2 \mu & 2 \nu-2 t
\end{array}\right) p_{k}=0
$$


Now, we can represent the system of the linear equations (63) - 651), 667) as a matrix equation $\tilde{\Gamma} \tilde{p}=0$ with $\tilde{p}=\left(p_{0}, \ldots, p_{k}\right) \in \mathbb{C}^{2 k+2}$ and the $(2 k+2) \times(2 k+2)$ matrix

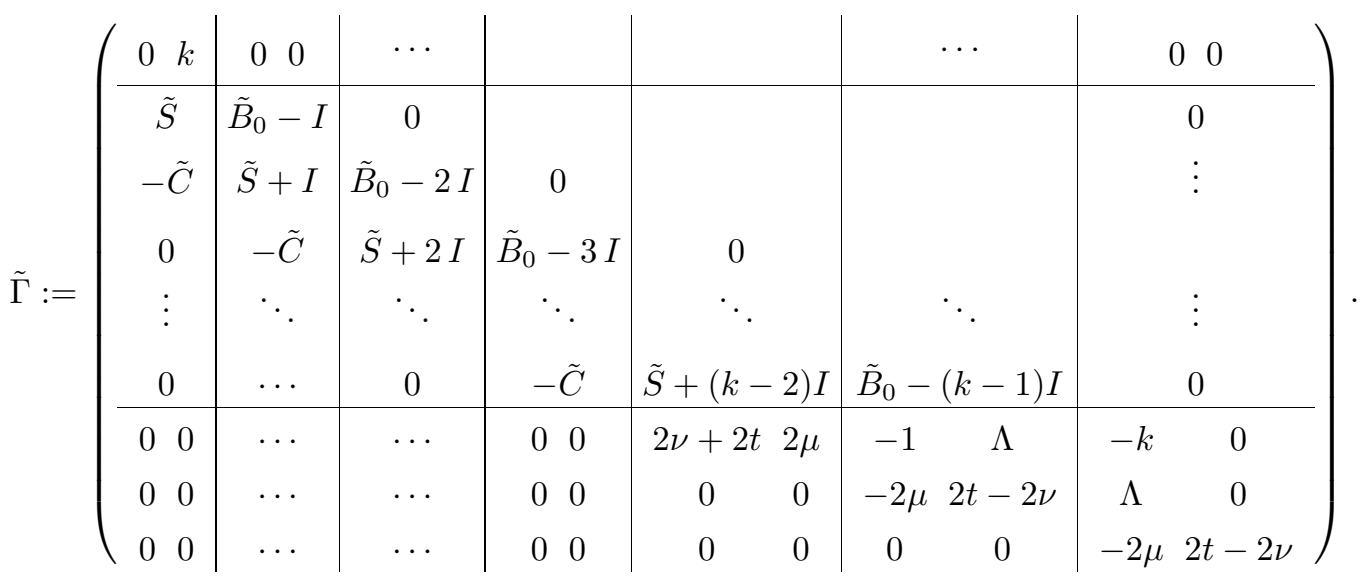

Let $\hat{\Gamma}$ be the $(2 k+1) \times(2 k+1)$-matrix obtained from $\tilde{\Gamma}$ by deleting the last row and column. Then $\lambda$ is a monodromy eigenvalue of $A(\kappa ; \mu, \nu)$ if and only if $0=\operatorname{det} \tilde{\Gamma}=(2 t-2 \nu) \operatorname{det} \hat{\Gamma}$, i.e., $\operatorname{det} \hat{\Gamma}=0$ since $\nu-t \neq 0$. Now, suppose that $\nu-t=0$. We will prove that also in this case $\lambda$ is a monodromy eigenvalue if and only if $\operatorname{det} \hat{\Gamma}=0$. Note that $\nu=t$ implies $\mu=0$, and therefore the equations in (66) are equivalent to

$$
\left(\begin{array}{cc}
0 & \Lambda \\
\Lambda & 0
\end{array}\right) p_{k}+\left(\begin{array}{cc}
4 t & 0 \\
0 & 0
\end{array}\right) p_{k-1}=0, \quad\left(\begin{array}{cc}
-4 t & 0 \\
0 & 0
\end{array}\right) p_{k}=0
$$

If $\lambda$ is a monodromy eigenvalue of $A(\kappa ; \mu, \nu)$, then the vector $\tilde{p}=\left(p_{0}, \ldots, p_{k}\right)$ is a nontrivial solution of the matrix equation $\tilde{\Gamma} \tilde{p}=0$ even though $T$ is not invertible for $\nu-t=0$. If we assume $\operatorname{det} \hat{\Gamma} \neq 0$, it follows that $\Lambda \neq 0$ and the first $2 k+1$ components of $\tilde{p}$ must be zero. In particular, $p_{1}=\cdots=p_{k-1}=0$, and the first equation in (69) yields $p_{k}=0$. Thus $\tilde{p}=0$, and this contradiction implies $\operatorname{det} \hat{\Gamma}=0$. Conversely, if $\operatorname{det} \hat{\Gamma}=0$, then either $\Lambda=0$ and $\tilde{p}:=e_{2 k+2}$ (the $2 k+2$-nd unit vector in $\mathbb{C}^{2 k+2}$ ) is a nontrivial solution of (63) - (65) and (69), or $\Lambda \neq 0$. In the latter case, there exists a vector $\hat{p} \neq 0$ with components $\hat{p}_{1}, \ldots, \hat{p}_{2 k+1} \in \mathbb{C}$ such that $\hat{\Gamma} \hat{p}=0$. Defining $q:=\frac{4 t}{\Lambda} \hat{p}_{2 k-1}$, then $\tilde{p}:=(\hat{p}, q) \in \mathbb{C}^{2 k+2}$ is a nontrivial solution of the equations (63) - 65) and (69), i.e., $\lambda$ is a monodromy eigenvalue. Hence, we have shown that for all $(\mu, \nu) \in \mathbb{C}^{2}$ with $\mu^{2} \neq \nu^{2}$ a point $\lambda \in \mathbb{C}$ is a monodromy eigenvalue of $A(\kappa ; \mu, \nu)$ if and only if $\operatorname{det} \hat{\Gamma}=0$. In order to prove that $\operatorname{det} \hat{\Gamma}$ is a polynomial in $\Lambda$ of degree $2 k-1$, we apply once more appropriate line transformations to $\hat{\Gamma}$. Adding successively the second to the fourth line, the fourth to the sixth line and so on up to the $2 k$-th line, then $\operatorname{det} \hat{\Gamma}=\operatorname{det} \Gamma$ with the $(2 k+1) \times(2 k+1)$-matrix

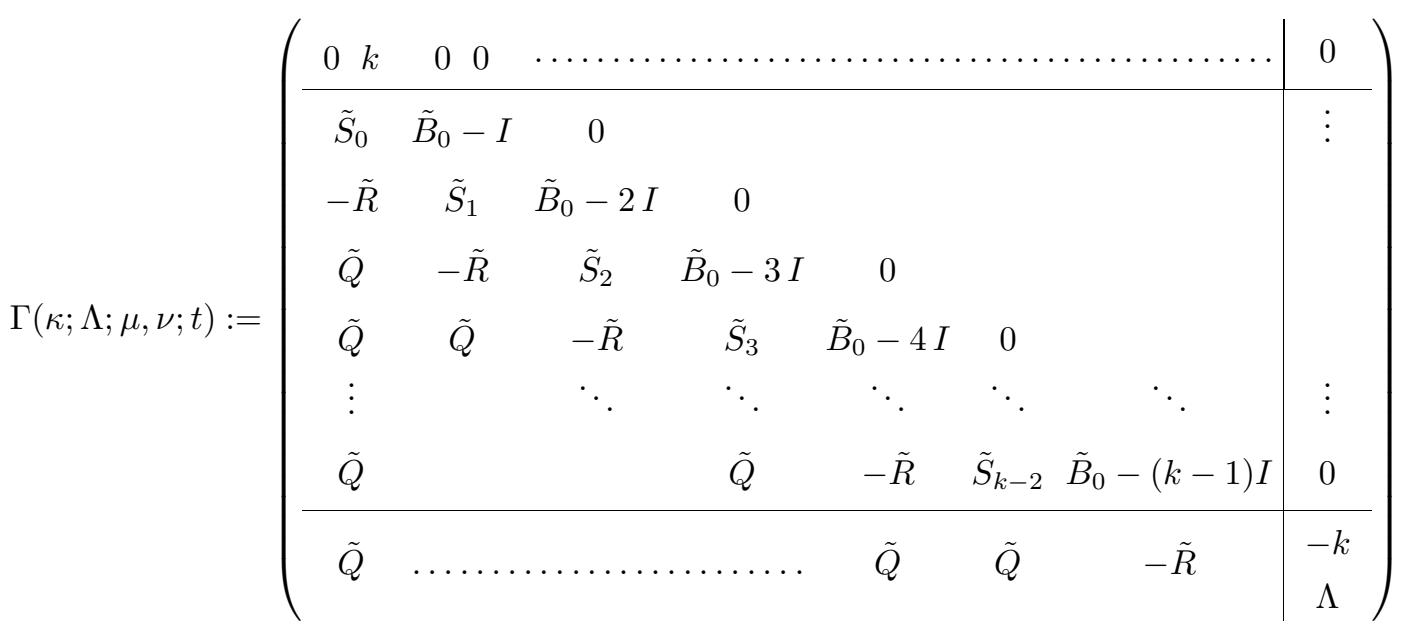

where

$$
\tilde{Q}:=\left(\begin{array}{cc}
-k & 0 \\
0 & 0
\end{array}\right), \quad \tilde{R}:=\left(\begin{array}{cc}
k & 0 \\
2 \mu & 2 \nu-2 t
\end{array}\right), \quad \tilde{S}_{n}:=\left(\begin{array}{cc}
-2 \nu-2 t-k & -2 \mu \\
2 \mu+\Lambda & 2 \nu-2 t+n-k
\end{array}\right), \quad n=0, \ldots, k-2 .
$$


Now, $\Lambda$ appears at most once in each row and each column, whereas only the first and the $2 k$-th line contain no entry involving $\Lambda$. It is easy to verify that $\operatorname{det} \Gamma(\Lambda ; \mu, \nu ; t)$ has the form $\pm k^{2} \Lambda^{2 k-1}+($ terms of lower order in $\Lambda)$, and therefore $\operatorname{det} \Gamma(\Lambda ; \mu, \nu ; t)$ is a polynomial in $\Lambda$ with polynomial coefficients in $\mu, \nu$ and $t$. Moreover, for all $(\mu, \nu) \in \mathbb{C}^{2}$ with $\mu^{2} \neq \nu^{2}$ a point $\lambda$ is a monodromy eigenvalue of $A(\kappa ; \mu, \nu)$ if and only if the determinant of $\Gamma(\kappa ; \Lambda ; \mu, \nu ; t)$ vanishes. As mentioned at the beginning of the proof, this result remains valid if we replace $t$ with $-t$. Hence, the zeros of the polynomials $\operatorname{det} \Gamma(\kappa ; \Lambda ; \mu, \nu ; t)$ and $\operatorname{det} \Gamma(\kappa ; \Lambda ; \mu, \nu ;-t)$ coincide, which implies that $\operatorname{det} \Gamma(\kappa ; \Lambda ; \mu, \nu ; t)=\operatorname{det} \Gamma(\kappa ; \Lambda ; \mu, \nu ;-t)$. Consequently, the polynomial $P(\kappa ; \lambda ; \mu, \nu):=\operatorname{det} \Gamma(\kappa ; \Lambda ; \mu, \nu ; t)$ contains no terms in $t$ of odd order, and the terms of even order in $t$ depend only on $t^{2}=\nu^{2}-\mu^{2}$. It follows that $P$ is a polynomial of degree $2 k-1$ in $\lambda$ with polynomial coefficients in $\mu$ and $\nu$, and the zeros of $P$ are exactly the monodromy eigenvalues of $A(\kappa ; \mu, \nu)$.

Next, we prove that the integers $1-k, \ldots, k-1$ are the zeros of the polynomial $P(\kappa ; \lambda ; 0,0)$. To this aim, let $\Gamma_{0}$ be the $(2 k \times 2 k)$ matrix obtained from $\hat{\Gamma}$ for $(\mu, \nu)=(0,0)$ by deleting the last row and column. Then

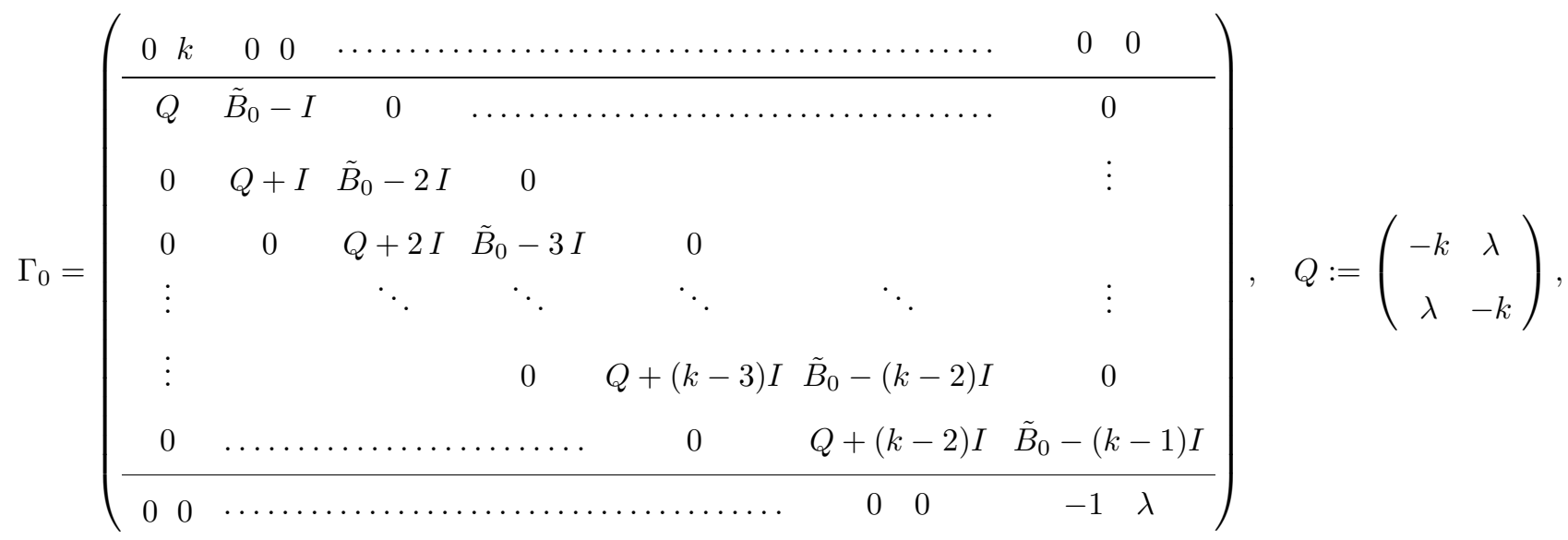

and $\operatorname{det} \hat{\Gamma}=\lambda \operatorname{det} \Gamma_{0}$. Moreover, $\operatorname{det} \Gamma_{0}=0$ if and only if the equation $\Gamma_{0}\left(p_{n}\right)_{n=0}^{k-1}=0$ has a nontrivial solution. Such a nontrivial solution is a constant multiple of the vector given by the recurrence formula

$$
\begin{aligned}
p_{0}:=\left(\begin{array}{c}
(k-1) ! \\
0
\end{array}\right), \quad p_{n} & =\left(n-\tilde{B}_{0}\right)^{-1}(Q+n-1) p_{n-1} \\
& =-\frac{1}{n(k-n)}\left(\begin{array}{cc}
(k-n)(k+1-n)-\lambda^{2} & \lambda \\
n \lambda & -n(k+1-n)
\end{array}\right) p_{n-1}
\end{aligned}
$$

for $n=1, \ldots, k-1$. By induction, it can be shown that

$$
p_{n}=(-1)^{n} \frac{(k-n-1) !}{n !} \prod_{j=1}^{n-1}\left[(k-j)^{2}-\lambda^{2}\right]\left(\begin{array}{c}
k(k-n)-\lambda^{2} \\
n \lambda
\end{array}\right) .
$$

Multiplying the vector $\left(p_{n}\right)_{n=0}^{k-1}$ from the left by the last line of $\Gamma_{0}$, we get

$$
0=(-1)^{k-1} \frac{1}{(k-1) !} \prod_{j=1}^{k-2}\left[(k-j)^{2}-\lambda^{2}\right]\left(-k+\lambda^{2}+(k-1) \lambda^{2}\right)=\frac{k(-1)^{k}}{(k-1) !} \prod_{j=1}^{k-1}\left[(k-j)^{2}-\lambda^{2}\right] .
$$

Hence, $\operatorname{det} \Gamma_{0}=0$ if and only if $\lambda^{2} \in\left\{1, \ldots,(k-1)^{2}\right\}$, and therefore $1-k, \ldots, k-1$ are the zeros of $P(\kappa ; \cdot ; 0,0)$.

It remains to deal with the case $k=1$, where we have to consider only the equations (60) and (62). Adding both equations in (62) to the second equation in (60) gives (65) with $p_{-1}:=0$. Hence, we can replace (60) - (62) with the linear system of equations consisting of the first equation in (63) and the equations (65), (66). Now, by applying a similar reduction procedure as in the case $k \geq 2$, we obtain the polynomial

$$
P\left(\frac{1}{2} ; \lambda ; \mu, \nu\right)=\operatorname{det}\left(\begin{array}{ccc}
0 & 1 & 0 \\
-1 & \Lambda & -1 \\
-2 \mu & 2 t-2 \nu & \Lambda
\end{array}\right)=\Lambda+2 \mu=\lambda+\mu
$$

whose zero $\lambda=-\mu$ is the uniquely determined monodromy eigenvalue of $A\left(\frac{1}{2} ; \mu, \nu\right)$ for each $(\mu, \nu) \in \mathbb{C}^{2}, \mu^{2} \neq \nu^{2}$. 
Corollary 1 For a fixed half-integer $\kappa=k-\frac{1}{2}$ with a positive integer $k$, there exists a neighbourhood $\mathfrak{U} \subset \mathbb{C}^{2}$ of $(0,0)$ such that $A(\kappa ; \mu, \nu)$ has exactly $2 k-1$ many monodromy eigenvalues $\lambda_{0}^{j}(\kappa ; \mu, \nu), j=1-k, \ldots, k-1$, for all $(\mu, \nu) \in \mathfrak{U}$ with $\mu^{2} \neq \nu^{2}$. Moreover, $\lambda_{0}^{j}(\kappa ; \mu, \nu)$ depends holomorphically on $(\mu, \nu)$, and $\lim _{(\mu, \nu) \rightarrow(0,0)} \lambda_{0}^{j}(\kappa ; \mu, \nu)=j$. In particular, monodromy eigenvalues and classical eigenvalues are distinct near $(\mu, \nu)=(0,0)$.

Remark 3 Monodromy eigenvalues also appear in the context of spheroidal wave equations. In [17, Sec. 3.534] they are characterised by a similar property as given in Lemma 5] but they are not specified in detail.

In view of Theorem 3 and Corollary 1 we could alternatively define the monodromy eigenvalues of $A(\kappa ; \mu, \nu)$ to be the zeros of the polynomial $P(\kappa ; \cdot ; \mu, \nu)$ for each $(\mu, \nu) \in \mathbb{C}^{2}$ (without the restriction $\mu^{2} \neq \nu^{2}$ ). Then the monodromy eigenvalues $\lambda_{0}^{j}(\kappa ; 0,0)=j, j=1-k, \ldots, k-1$, fill in the gap of integers appearing in the spectrum of $A(\kappa ; 0,0)$. Moreover, $P\left(\frac{1}{2} ; \lambda ; \mu, \nu\right)=\lambda+\mu$ is just the polynomial given by Lemma 4 in the case $N=1$, and its zero $\lambda_{0}^{0}\left(\frac{1}{2} ; \mu, \nu\right)=-\mu$ satisfies the partial differential equation (29) for $\kappa=\frac{1}{2}$. In the next section we prove that the monodromy eigenvalues of $A(\kappa ; \mu, \nu)$ are solutions of the PDE (29) for each half-integer $\kappa \in\left\{\frac{1}{2}, \frac{3}{2}, \frac{5}{2}, \ldots\right\}$.

\section{MONODROMY PRESERVING DEFORMATIONS}

In [9], 10] and [1], Jimbo, Miwa \& Ueno developed a general theory for monodromy preserving deformations of linear ordinary differential equations with rational coefficients. As a main result, they proved that the monodromy data (Stokes multipliers, connection matrices and exponents of formal monodromy) do not depend on the deformation parameters if and only if certain non-linear differential equations, the so-called deformation equations, are satisfied. This result, however, was proved under the restriction that the characteristic values at regular singular points do not differ by an integer. On the other hand, in the theory of special functions and in many physical applications the case where the characteristic values differ by an integer is of great significance. In this section we consider the isomonodromy problem for linear systems with two fixed regular singular points and coefficients which depend on one parameter $t$. Assuming that the characteristic values at the singular points are distinct and independent of $t$, we will show that certain components of the monodromy data are constant with respect to $t$ if a deformation equation of the type [9, (1.18)] is satisfied. Since the monodromy components in question determine the existence of solutions of the form (54) and (55), they are relevant to monodromy and classical eigenvalue problems. Applying the results to the system (11) with an eigenvalue $t$ of $C$ as deformation parameter, it finally turns out that the deformation equation is in principle the characteristic equation of the partial differential equation (29).

We start with some basic facts about parameter-dependent regular singular systems. At first, let us consider a family of $(2 \times 2)$ systems of differential equations

$$
\frac{\partial y}{\partial x}(x, t)=\Phi(x, t) y(x, t), \quad(x, t) \in(\mathfrak{B} \backslash\{0\}) \times \mathfrak{D},
$$

in an open disc $\mathfrak{B} \subset \mathbb{C}$ with centre 0 that depends on a parameter $t$ varying in some real or complex domain $\mathfrak{D}$. It is assumed that (70) has a regular singular point at 0 for all $t \in \mathfrak{D}$. More precisely, we suppose that the coefficient matrix $\Phi$ of (70) has the following properties:

(a) $\Phi(x, t)=\frac{1}{x} \Psi(x, t)$, where $\Psi: \mathfrak{B} \times \mathfrak{D} \longrightarrow \mathrm{M}_{2}(\mathbb{C})$ is an analytical matrix function.

(b) The eigenvalues $\alpha$ and $\beta$ of $\Psi(0, t)$ are distinct and independent of $t \in \mathfrak{D} ;$ moreover, $\operatorname{Re} \alpha \leq \operatorname{Re} \beta$.

(c) There is an analytical function $G: \mathfrak{D} \longrightarrow \mathrm{M}_{2}(\mathbb{C})$ such that $G(t)$ is invertible and

$$
G(t)^{-1} \Psi(0, t) G(t)=\operatorname{diag}(\alpha, \beta)=: D, \quad t \in \mathfrak{D},
$$

Note that such a matrix function $G$ always exists since the eigenvalues of $\Psi(0, t)$ are distinct (see [24, Chap. VII, Sec. 25, Theorem 25.1]).

Lemma 6 If the conditions (a) - (c) are satisfied, then the system (70) has a fundamental matrix of the form

$$
Y(x, t)=G(t) H(x, t) x^{D} x^{J(t)}
$$

where $H: \mathfrak{B} \times \mathfrak{D} \longrightarrow \mathrm{M}_{2}(\mathbb{C})$ is analytic, $H(0, t)=I$ for all $t \in \mathfrak{D}$, and

$$
J(t)=\left(\begin{array}{cc}
0 & 0 \\
p(t) & 0
\end{array}\right)
$$

with some analytical function $p: \mathfrak{D} \longrightarrow \mathbb{C}$. Moreover, if $\beta-\alpha$ is not an integer, then $p \equiv 0$. 
Proof. If $\beta-\alpha$ is not an integer, the existence of such a fundamental matrix with $J \equiv 0$ is well known cf. [20]. Hence, we have to consider only the case that $k:=\beta-\alpha$ is a positive integer. By the transformation

$$
y(x, t)=x^{\alpha} G(t) y_{0}(x, t),
$$

the system (70) is equivalent to the differential equation

$$
x \frac{\partial y_{0}}{\partial x}(x, t)=\Psi_{0}(x, t) y_{0}(x, t), \quad(x, t) \in(\mathfrak{B} \backslash\{0\}) \times \mathfrak{D},
$$

where $\Psi_{0}(x, t):=G(t)^{-1} \Psi(x, t) G(t)-\alpha$ is an analytical matrix function,

$$
\Psi_{0}(x, t)=\sum_{n=0}^{\infty} x^{n} \Psi_{0, n}(t), \quad(x, t) \in \mathfrak{B} \times \mathfrak{D},
$$

with $\Psi_{0,0}(t)=\operatorname{diag}(0, k)$ for all $t \in \mathfrak{D}$. Now, for $j=1, \ldots, k-1$ we recursively apply the transformations

$$
y_{j-1}(x, t)=\left(\begin{array}{cc}
1 & 0 \\
\frac{x}{j-k} \psi_{j-1}(t) & x
\end{array}\right) y_{j}(x, t),
$$

where $\psi_{j-1}$ denotes the $(2,1)$-coefficient of the matrix $\Psi_{j-1,1}$. At each step, $y_{j}(x, t)$ is a solution of a system

$$
x \frac{\partial y_{j}}{\partial x}(x, t)=\Psi_{j}(x, t) y_{j}(x, t), \quad(x, t) \in(\mathfrak{B} \backslash\{0\}) \times \mathfrak{D},
$$

where the coefficient matrix $\Psi_{j}$ is analytic in $\mathfrak{B} \times \mathfrak{D}$,

$$
\Psi_{j}(x, t)=\sum_{n=0}^{\infty} x^{n} \Psi_{j, n}(t), \quad(x, t) \in \mathfrak{B} \times \mathfrak{D},
$$

with $\Psi_{j, 0}(t)=\operatorname{diag}(0, k-j)$ for all $t \in \mathfrak{D}$, and $\Psi_{j, n}(t), n=1, \ldots, j-1$, are lower triangular matrix functions (that means, the $(1,2)$ component is identically zero). Finally, by the shearing transformation

$$
y_{k-1}(x, t)=\left(\begin{array}{ll}
1 & 0 \\
0 & x
\end{array}\right) y_{k}(x, t),
$$

we obtain a differential equation

$$
x \frac{\partial y_{k}}{\partial x}(x, t)=\Psi_{k}(x, t) y_{k}(x, t), \quad(x, t) \in(\mathfrak{B} \backslash\{0\}) \times \mathfrak{D},
$$

where $\Psi_{k}: \mathfrak{B} \times \mathfrak{D} \longrightarrow \mathrm{M}_{2}(\mathbb{C})$ is an analytical matrix function,

$$
\Psi_{k}(x, t)=\sum_{n=0}^{\infty} x^{n} \Psi_{k, n}(t), \quad(x, t) \in \mathfrak{B} \times \mathfrak{D},
$$

satisfying

$$
\Psi_{k, 0}(t)=\left(\begin{array}{cc}
0 & 0 \\
p(t) & 0
\end{array}\right)=: J(t), \quad t \in \mathfrak{D},
$$

with some analytical function $p: \mathfrak{D} \longrightarrow \mathbb{C}$. Note that $p$ is just the $(2,1)$-component of $\Psi_{k-1,1}$. Moreover, $\Psi_{k, n}(t)$, $n=0, \ldots, k$, are lower triangular matrices for all $t \in \mathfrak{D}$. Now, the system (77) has a fundamental matrix of the form

$$
\tilde{Y}(x, t)=\tilde{H}(x, t) x^{J(t)}
$$

provided that $\tilde{H}$ is a solution of the matrix differential equation

$$
x \frac{\partial \tilde{H}}{\partial x}(x, t)=\Psi_{k}(x, t) \tilde{H}(x, t)-\tilde{H}(x, t) J(t), \quad(x, t) \in \mathfrak{B} \times \mathfrak{D},
$$


such that for each $t \in \mathfrak{D}$ the matrix $\tilde{H}(x, t)$ is invertible for some, and hence all, $x \in \mathfrak{B}$. Obviously, (78) has a formal solution

$$
\tilde{H}(x, t)=\sum_{n=0}^{\infty} x^{n} \tilde{H}_{n}(t), \quad(x, t) \in \mathfrak{B} \times \mathfrak{D}
$$

where $\tilde{H}_{0}(t)=I$ and the coefficients $\tilde{H}_{n}, n>0$, are uniquely determined by the recurrence relation

$$
(J(t)-n) \tilde{H}_{n}(t)-\tilde{H}_{n}(t) J(t)=-\sum_{j=0}^{n-1} \Psi_{k, n-j}(t) \tilde{H}_{j}(t)
$$

Following the proof of Theorem 5.3 in the book of Wasow 24], it can be shown that the series (79) converges uniformly in every compact subset of $\mathfrak{B} \times \mathfrak{D}$. Thus, a Weierstrass theorem implies that $\tilde{H}$ is analytic in $\mathfrak{B} \times \mathfrak{D}$, and therefore $\tilde{H}$ is an actual solution of (78). Further, since $J(t)$ has the special form (171) and $\Psi_{k, j}(t), j=0, \ldots, k$, are lower triangular matrices, it is easy to verify that $\tilde{H}_{j}(t)$ are lower triangular matrices for $j=0, \ldots, k$. Now, by combining the transformations (72), (74) and (76), it follows that the differential equation (70) has a fundamental matrix of the form

$$
Y(x, t)=x^{\alpha} G(t)\left(\begin{array}{cc}
1 & 0 \\
x q(x, t) & x^{k}
\end{array}\right) \tilde{H}(x, t) x^{J(t)}
$$

where $q(x, t)$ is a polynomial in $x$ of degree $n-1$ with coefficients depending analytically on $t$, and $\tilde{H}(x, t)$ is an analytical matrix function of the type

$$
\tilde{H}(x, t)=\left(\begin{array}{cc}
h_{11}(x, t) & x^{k+1} h_{12}(x, t) \\
h_{21}(x, t) & h_{22}(x, t)
\end{array}\right)
$$

satisfying $h_{11}(0, t)=h_{22}(0, t)=1$. Now, if we define

$$
H(x, t):=\left(\begin{array}{cc}
h_{11}(x, t) & x h_{12}(x, t) \\
x q(x, t) h_{11}(x, t)+x^{k+1} h_{21}(t) & x^{2} q(x, t) h_{12}(x, t)+h_{22}(x, t)
\end{array}\right),
$$

then $H: \mathfrak{B} \times \mathfrak{D} \longrightarrow \mathrm{M}_{2}(\mathbb{C})$ is analytic, $H(0, t)=I$ for all $t \in \mathfrak{D}$, and

$$
\left(\begin{array}{cc}
1 & 0 \\
x q(x, t) & x^{k}
\end{array}\right) \tilde{H}(x, t)=H(x, t)\left(\begin{array}{cc}
1 & 0 \\
0 & x^{k}
\end{array}\right) .
$$

Hence, we can write the fundamental matrix (81) in the form $Y(x, t)=G(t) H(x, t) x^{D} x^{J(t)}$, where $H$ has the properties stated in the Lemma.

Now, we consider a family of $(2 \times 2)$ differential systems

$$
\frac{\partial y}{\partial x}(x, t)=\Phi(x, t) y(x, t), \quad(x, t) \in(\mathfrak{G} \backslash\{0,1\}) \times \mathfrak{D}
$$

in a domain $\mathfrak{G}, \mathfrak{B}_{0} \cup \mathfrak{B}_{1} \subset \mathfrak{G} \subset \mathbb{C}$, with regular singular points at $x=0$ and $x=1$ and a parameter $t$ varying in some domain $\mathfrak{D} \subset \mathbb{R}$ or $\mathfrak{D} \subset \mathbb{C}$. Further, we assume that the coefficient matrix $\Phi$ in (82) has the form

$$
\Phi(x, t)=\frac{1}{x(x-1)} \Psi(x, t), \quad(x, t) \in(\mathfrak{G} \backslash\{0,1\}) \times \mathfrak{D}
$$

where $\Psi: \mathfrak{G} \times \mathfrak{D} \longrightarrow \mathrm{M}_{2}(\mathbb{C})$ is an analytical matrix function with the following properties:

(I) The eigenvalues $\alpha, \beta$ of $\Psi(a, t)$ are distinct and independent of $t \in \mathfrak{D}$ and $a \in\{0,1\}$; in addition, Re $\alpha \leq \operatorname{Re} \beta$.

(II) There are analytical functions $G_{a}: \mathfrak{D} \longrightarrow \mathrm{M}_{2}(\mathbb{C}), a \in\{0,1\}$, such that $G_{a}(t)$ is invertible for all $t \in \mathfrak{D}$ and

$$
G_{a}(t)^{-1} \Psi(a, t) G_{a}(t)=(-1)^{a} \operatorname{diag}(\alpha, \beta) .
$$


From Lemma 6] it follows that the system (82) possesses a fundamental matrix of the form

$$
Y_{a}(x, t)=G_{a}(t) H_{a}(x, t)(x-a)^{D}(x-a)^{J_{a}(t)}
$$

in the unit $\operatorname{disc} \mathfrak{B}_{a} \subset \mathfrak{G}$ with centre $a \in\{0,1\}$, where $H_{a}: \mathfrak{B}_{a} \times \mathfrak{D} \longrightarrow \mathrm{M}_{2}(\mathbb{C})$ is an analytical matrix function satisfying $H_{a}(0, t)=I$ for all $t \in \mathfrak{D}, D=\operatorname{diag}(\alpha, \beta)$, and

$$
J_{a}(t)=\left(\begin{array}{cc}
0 & 0 \\
p_{a}(t) & 0
\end{array}\right)
$$

with some analytical function $p_{a}: \mathfrak{D} \longrightarrow \mathbb{C}$. By analytic continuation along curves, we can assume that $Y_{a}$ is defined on the universal covering $\mathfrak{R}$ of the set $\mathfrak{G} \backslash\{0,1\}$. Since $Y_{a}\left(x e^{2 i \pi}+a, t\right)=Y_{a}(x+a, t) e^{2 i \pi D}\left[I+2 \pi i J_{a}(t)\right]$ for all $(x, t) \in\left(\mathfrak{B}_{0} \backslash\{0\}\right) \times \mathfrak{D}$, the diagonal matrix $D$ and the Jordan type matrix $J_{a}(t)$ represent the monodromy behaviour of $Y_{a}$ corresponding to a circuit around $a \in\{0,1\}$. Moreover, as $Y_{0}$ and $Y_{1}$ are both fundamental matrices of the same differential equation (82), there exists an analytical matrix function $Q: \mathfrak{D} \longrightarrow \mathrm{M}_{2}(\mathbb{C})$ such that $Y_{0}(x, t)=Y_{1}(x, t) Q(t)$ for all $(x, t) \in(\mathfrak{G} \backslash\{0,1\}) \times \mathfrak{D}$, which is called the connection matrix for $Y_{0}$ and $Y_{1}$. The next result gives a sufficient condition that certain components of the monodromy data $J_{a}$ and $Q$ are constant in $\mathfrak{D}$. For this reason, we establish in addition to (I) - (II) the following assumptions on the coefficient matrix $\Phi$ :

(III) There exists an analytical function $\Omega: \mathfrak{G} \times \mathfrak{D} \longrightarrow \mathrm{M}_{2}(\mathbb{C})$ such that

$$
\frac{\partial \Phi}{\partial t}(x, t)+\Phi(x, t) \Omega(x, t)=\Omega(x, t) \Phi(x, t)+\frac{\partial \Omega}{\partial x}(x, t), \quad(x, t) \in(\mathfrak{G} \backslash\{0,1\}) \times \mathfrak{D},
$$

(IV) The matrix functions $G_{a}, a \in\{0,1\}$, satisfy the linear differential equations

$$
\frac{\partial G_{a}}{\partial t}(t)=\Omega(a, t) G_{a}(t), \quad t \in \mathfrak{D} .
$$

Theorem 4 If the conditions (I) - (IV) are satisfied, then

$$
\frac{\partial J_{a}}{\partial t} \equiv \frac{\partial Q_{21}}{\partial t} \equiv 0 \text { in } \mathfrak{D}
$$

where the Jordan matrices $J_{a}, a \in\{0,1\}$, are given by (84) and $Q_{12}: \mathfrak{D} \longrightarrow \mathbb{C}$ denotes the $(1,2)$-component of the connection matrix $Q$ for $Y_{0}$ and $Y_{1}$.

Proof. Let $\gamma:=\beta-\alpha$, and for fixed $a \in\{0,1\}$ we define

$$
Z_{a}(x, t):=\frac{\partial Y_{a}}{\partial t}(x, t)-\Omega(x, t) Y_{a}(x, t), \quad(x, t) \in \mathfrak{R} \times \mathfrak{D} .
$$

From (82) and the deformation equation (85) it follows that

$$
\begin{aligned}
\frac{\partial Z_{a}}{\partial x} & =\frac{\partial^{2} Y_{a}}{\partial x \partial t}-\frac{\partial \Omega}{\partial x} Y_{a}-\Omega \frac{\partial Y_{a}}{\partial x}=\frac{\partial\left(\Phi Y_{a}\right)}{\partial t}-\frac{\partial \Omega}{\partial x} Y_{a}-\Omega \Phi Y_{a} \\
& =\Phi \frac{\partial Y_{a}}{\partial t}+\left(\frac{\partial \Phi}{\partial t}-\frac{\partial \Omega}{\partial x}-\Omega \Phi\right) Y_{a}=\Phi\left(\frac{\partial Y_{a}}{\partial t}-\Omega Y_{a}\right)=\Phi Z_{a}
\end{aligned}
$$

and therefore $Z_{a}$ is a matrix solution of the differential equation (82) in $\mathfrak{R}$. Hence, there exists an analytical function $C_{a}: \mathfrak{D} \longrightarrow \mathrm{M}_{2}(\mathbb{C})$ such that

$$
Z_{a}(x, t)=Y_{a}(x, t) C_{a}(t), \quad(x, t) \in \mathfrak{R} \times \mathfrak{D}
$$

Now, by means of the differential equation (86), we get

$$
\begin{aligned}
\frac{\partial Y_{a}}{\partial t} & =\frac{\partial G_{a}}{\partial t} H_{a}(x-a)^{D}(x-a)^{J_{a}}+G_{a} \frac{\partial H_{a}}{\partial t}(x-a)^{D}(x-a)^{J_{a}}+\log (x-a) G_{a} H_{a}(x-a)^{D} \frac{\partial J_{a}}{\partial t}(x-a)^{J_{a}} \\
& =\left(\Omega(a, \cdot) G_{a} H_{a}+G_{a} \frac{\partial H_{a}}{\partial t}+(x-a)^{\gamma} \log (x-a) G_{a} H_{a} \frac{\partial J_{a}}{\partial t}\right)(x-a)^{D}(x-a)^{J_{a}}
\end{aligned}
$$


and since $C_{a}=Y_{a}^{-1} Z_{a}$, it results that

$$
\begin{aligned}
(x-a)^{D}(x-a)^{J_{a}} & C_{a}(x-a)^{-J_{a}}(x-a)^{-D} \\
& =(x-a)^{D}(x-a)^{J_{a}} Y_{a}^{-1}\left(\frac{\partial Y_{a}}{\partial t}-\Omega Y_{a}\right)(x-a)^{-J_{a}}(x-a)^{-D} \\
& =H_{a}^{-1} G_{a}^{-1}(\Omega(a, \cdot)-\Omega) G_{a} H_{a}+H_{a}^{-1} \frac{\partial H_{a}}{\partial t}+(x-a)^{\gamma} \log (x-a) \frac{\partial J_{a}}{\partial t} \\
& =(x-a) F_{a}+(x-a)^{\gamma} \log (x-a)\left(\begin{array}{cc}
0 & 0 \\
\frac{\partial p_{a}}{\partial t} & 0
\end{array}\right)
\end{aligned}
$$

with some analytical function $F_{a}: \mathfrak{G} \times \mathfrak{D} \longrightarrow \mathrm{M}_{2}(\mathbb{C})$. Further, by setting

$$
C_{a}(t)=:\left(\begin{array}{ll}
C_{11}(t) & C_{12}(t) \\
C_{21}(t) & C_{22}(t)
\end{array}\right), \quad t \in \mathfrak{D},
$$

(for clarity, we omit the index $a$ in the entries of $C_{a}$ ), we have

$$
\begin{aligned}
(x-a)^{D} & (x-a)^{J_{a}} C_{a}(x-a)^{-J_{a}}(x-a)^{-D} \\
= & \left(\begin{array}{cc}
1 & 0 \\
(x-a)^{\gamma} \log (x-a) p_{a} & (x-a)^{\gamma}
\end{array}\right)\left(\begin{array}{cc}
C_{11} & C_{12} \\
C_{21} & C_{22}
\end{array}\right)\left(\begin{array}{cc}
1 & 0 \\
-\log (x-a) p_{a} & (x-a)^{-\gamma}
\end{array}\right) \\
= & \left(\begin{array}{cc}
C_{11}-\log (x-a) p_{a} C_{12} & (x-a)^{-\gamma} C_{12} \\
* & C_{22}+\log (x-a) p_{a} C_{12}
\end{array}\right) .
\end{aligned}
$$

Comparing (88) to (89), it follows that $C_{12} \equiv 0$ in $\mathfrak{D}$ since the function in (88) is bounded at $x=a$. This in turn implies $C_{11} \equiv C_{22} \equiv 0$ as the diagonal entries in (88) have a zero at $x=a$ for all $t \in \mathfrak{D}$. Finally, we obtain that

$$
(x-a)^{D}(x-a)^{J_{a}} C_{a}(x-a)^{-J_{a}}(x-a)^{-D}=\left(\begin{array}{cc}
0 & 0 \\
(x-a)^{\gamma} C_{21} & 0
\end{array}\right)
$$

has no logarithmic singularity at $x=a$ and therefore the last term (88) vanishes identically. Hence, $\frac{\partial J_{a}}{\partial t} \equiv 0$ in $\mathfrak{D}$.

Next, we prove that $\frac{\partial Q_{12}}{\partial t} \equiv 0$. Since $Y_{0}(x, t)=Y_{1}(x, t) Q(t)$, it follows that

$$
\frac{\partial Y_{0}}{\partial t}=\frac{\partial Y_{1}}{\partial t} Q+Y_{1} \frac{\partial Q}{\partial t}
$$

Further, from $Z_{a}(x, t)=Y_{a}(x, t) C_{a}(t)$ we get

$$
\frac{\partial Y_{0}}{\partial t} Y_{0}^{-1}-\Omega=Y_{0} C_{0} Y_{0}^{-1}, \quad \frac{\partial Y_{1}}{\partial t} Y_{1}^{-1}-\Omega=Y_{1} C_{1} Y_{1}^{-1} .
$$

By means of (91) and $Y_{0}^{-1}=Q^{-1} Y_{1}^{-1}$, the first equation in (92) becomes

$$
\frac{\partial Y_{1}}{\partial t} Y_{1}^{-1}-\Omega=Y_{1} Q C_{0} Q^{-1} Y_{1}^{-1}-Y_{1} \frac{\partial Q}{\partial t} Q^{-1} Y_{1}^{-1}
$$

Now, 93) and the second equation in (92) imply

$$
Y_{1} C_{1} Y_{1}^{-1}=Y_{1} Q C_{0} Q^{-1} Y_{1}^{-1}-Y_{1} \frac{\partial Q}{\partial t} Q^{-1} Y_{1}^{-1}
$$

and therefore

$$
\frac{\partial Q}{\partial t}=Q C_{0}-C_{1} Q
$$

Note that the matrix function $C_{a}$ has the form

$$
C_{a}(t)=\left(\begin{array}{cc}
0 & 0 \\
c_{a}(t) & 0
\end{array}\right), \quad a \in\{0,1\}
$$


Hence, if we set

$$
Q(t)=:\left(\begin{array}{ll}
Q_{11}(t) & Q_{12}(t) \\
Q_{21}(t) & Q_{22}(t)
\end{array}\right), \quad t \in \mathfrak{D}
$$

then (94) is equivalent to the system

$$
\frac{\partial}{\partial t}\left(\begin{array}{ll}
Q_{11} & Q_{12} \\
Q_{21} & Q_{22}
\end{array}\right)=\left(\begin{array}{cc}
c_{0} Q_{12} & 0 \\
c_{0} Q_{22}-c_{1} Q_{11} & c_{1} Q_{12}
\end{array}\right)
$$

and we immediately obtain that $\frac{\partial Q_{12}}{\partial t} \equiv 0$ in $\mathfrak{D}$.

In the following we apply the results of Lemma 6 and Theorem 4 to a family of $(2 \times 2)$ differential systems

$$
\frac{\partial y}{\partial x}(x, t)=\left[\frac{1}{x} B_{0}(t)+\frac{1}{x-1} B_{1}(t)+C(t)\right] y(x, t), \quad(x, t) \in(\mathbb{C} \backslash\{0,1\}) \times \mathfrak{D},
$$

where $t \in \mathfrak{D}$ with some domain $\mathfrak{D} \subset \mathbb{R}$ or $\mathfrak{D} \subset \mathbb{C}$, and the coefficients $B_{0}, B_{1}, C: \mathfrak{D} \longrightarrow \mathrm{M}_{2}(\mathbb{C})$ are supposed to be analytical functions. Further, we assume that the following conditions hold:

(i) The eigenvalues $\alpha, \beta$ of $B_{0}(t)$ are distinct and independent of $t \in \mathfrak{D}$. Moreover, they coincide with the eigenvalues of $B_{1}(t)$, and $\operatorname{Re} \alpha \leq \operatorname{Re} \beta$.

(ii) There are analytical functions $G_{a}: \mathfrak{D} \longrightarrow \mathrm{M}_{2}(\mathbb{C}), a \in\{0,1\}$, such that $G_{a}(t)$ is invertible and

$$
G_{0}(t)^{-1} B_{0}(t) G_{0}(t)=-G_{1}(t)^{-1} B_{1}(t) G_{1}(t)=\operatorname{diag}(\alpha, \beta)=: D, \quad t \in \mathfrak{D}
$$

Let $Y_{a}, a \in\{0,1\}$, be fundamental matrices of 195 in the open disc $\mathfrak{B}_{a} \subset \mathbb{C}$ with centre $a$ and radius 1 having the form (83), where $H_{a}: \mathfrak{B} \times \mathfrak{D} \longrightarrow \mathrm{M}_{2}(\mathbb{C})$ is analytical, $H_{a}(0, t)=I$ for all $t \in \mathfrak{D}$, and $J_{a}(t)$ is given by (84) with some analytical function $p_{a}: \mathfrak{D} \longrightarrow \mathbb{C}$. Again, by analytic continuation, we assume that $Y_{a}$ is defined on the universal covering $\mathfrak{R}$ of $\mathbb{C} \backslash\{0,1\}$, and we denote by $Q: \mathfrak{D} \longrightarrow \mathrm{M}_{2}(\mathbb{C})$ the connection matrix for $Y_{0}$ and $Y_{1}$. In the sequel we are looking for conditions such that for fixed $t \in \mathfrak{D}$ the system (95) has one of the following properties:

(P) There exists a fundamental matrix $Y$ of the form

$$
Y(x)=[x(1-x)]^{\alpha} P(x) e^{C(t) x}, \quad x \in \mathbb{C} \backslash\{0,1\},
$$

where $P: \mathbb{C} \longrightarrow \mathrm{M}_{2}(\mathbb{C})$ is a polynomial matrix function.

(H) There exists a nontrivial solution $y$ of the form

$$
y(x)=[x(1-x)]^{\beta} h(x), \quad x \in \mathbb{C} \backslash\{0,1\},
$$

where $h: \mathbb{C} \longrightarrow \mathbb{C}^{2}$ is an entire vector function.

Lemma 7 Suppose that the conditions (i) and (ii) are satisfied, and let $t \in \mathfrak{D}$. Then the system (95) has the property $(\mathrm{P})$ if and only if $\beta-\alpha$ is an integer and $p_{0}(t)=p_{1}(t)=0$, and it has the property $(\mathrm{H})$ if and only if $Q_{21}(t)=0$.

Proof. By means of the transformation $y(x)=x^{\alpha}(x-1)^{\alpha} \tilde{y}(x)$, the differential equation (95) is equivalent to the system

$$
\frac{\partial \tilde{y}}{\partial x}(x, t)=\left[\frac{1}{x} \tilde{B}_{0}(t)+\frac{1}{x-1} \tilde{B}_{1}(t)+C(t)\right] \tilde{y}(x, t), \quad(x, t) \in(\mathbb{C} \backslash\{0,1\}) \times \mathfrak{D}
$$

where $\tilde{B}_{0}(t):=B_{0}(t)-\alpha$ and $\tilde{B}_{1}(t):=B_{1}(t)-\alpha$. Moreover,

$$
\tilde{Y}_{a}(x, t)=G_{a}(t) H_{a}(x, t)\left(\begin{array}{cc}
1 & 0 \\
0 & (x-a)^{\beta-\alpha}
\end{array}\right)(x-a)^{J_{a}(t)}
$$

are fundamental matrices of (98) in a neighbourhood of $a \in\{0,1\}$. First, suppose that $\beta-\alpha$ is an integer and that $p_{0}(t)=p_{1}(t)=0$ holds. In this case $J_{0}(t)=J_{1}(t)=0$, and the system (98) has a holomorphic fundamental matrix 
since $(x-a)^{\beta-\alpha}$ is holomorphic and $\tilde{Y}_{0}(\cdot, t), \tilde{Y}_{1}(\cdot, t)$ contain no logarithmic terms. Moreover, as the coefficient matrix $\Phi(\cdot, t)$ of (95) is a rational function which is bounded at infinity, the extension of Halphen's Theorem 6 , Theorem $2.4]$ implies that the system (98) has a fundamental matrix of the form $\tilde{Y}(x)=R(x) e^{C(t) x}$ with some rational (and hence polynomial) matrix function $R: \mathbb{C} \longrightarrow \mathrm{M}_{2}(\mathbb{C})$. Conversely, if (95) has a fundamental matrix of the form (96), then $\tilde{Y}_{0}(\cdot, t)$ and $\tilde{Y}_{1}(\cdot, t)$ are holomorphic matrix functions, which gives $\beta-\alpha \in \mathbb{Z}$ and $J_{0}(t)=J_{1}(t)=0$.

Next, let us assume that $Q_{12}(t)=0$. If we define

$$
y(x):=Y_{0}(x, t)\left(\begin{array}{l}
0 \\
1
\end{array}\right)=x^{\beta} G_{0}(t) H_{0}(x, t)\left(\begin{array}{l}
0 \\
1
\end{array}\right),
$$

then $y$ is a nontrivial solution of (95), and $x^{-\beta} y(x)$ is analytic at $x=0$. Since $Y_{0}(x, t)=Y_{1}(x, t) Q(t)$ and $Q_{12}(t)=0$, we obtain

$$
y(x)=Y_{1}(x, t) Q(t)\left(\begin{array}{l}
0 \\
1
\end{array}\right)=(x-1)^{\beta} G_{1}(t) H_{1}(x, t)\left(\begin{array}{c}
0 \\
Q_{22}(t)
\end{array}\right),
$$

and therefore $(1-x)^{-\beta} y(x)$ is analytic in a neighbourhood of $x=1$. Now, by the existence and uniqueness theorem, $h(x):=[x(1-x)]^{-\beta} y(x)$ can be extended to an entire vector function. Conversely, suppose that (95) has a nontrivial solution of the form (97). Then

$$
y(x)=Y_{0}(x, t)\left(\begin{array}{c}
0 \\
c_{0}
\end{array}\right)=Y_{1}(x, t)\left(\begin{array}{c}
0 \\
c_{1}
\end{array}\right)
$$

with some constants $c_{0}, c_{1} \in \mathbb{C} \backslash\{0\}$. Since $Y_{0}(x, t)=Y_{1}(x, t) Q(t)$, it follows that

$$
Q(t)\left(\begin{array}{c}
0 \\
c_{0}
\end{array}\right)=\left(\begin{array}{c}
0 \\
c_{1}
\end{array}\right)
$$

which gives $Q_{21}(t)=0$.

Now, in addition to (i) - (ii), we assume that the coefficients of (95) satisfy the following conditions:

(iii) There exists an analytical function $\Omega: \mathbb{C} \times \mathfrak{D} \longrightarrow \mathrm{M}_{2}(\mathbb{C})$ such that the deformation equation (85) holds in $(\mathbb{C} \backslash\{0,1\}) \times \mathfrak{D}$, where $\Phi$ is given by

$$
\Phi(x, t):=\frac{1}{x} B_{0}(t)+\frac{1}{x-1} B_{1}(t)+C(t), \quad(x, t) \in(\mathbb{C} \backslash\{0,1\}) \times \mathfrak{D} .
$$

(iv) The matrix functions $G_{a}, a \in\{0,1\}$, satisfy the differential equations

$$
\frac{\partial G_{a}}{\partial t}(t)=\Omega(a, t) G_{a}(t), \quad t \in \mathfrak{D}
$$

The next result is an immediate consequence of Theorem 4 and Lemma 7.

Corollary 2 Suppose that the conditions (i) - (iv) are satisfied. If (P) holds for one $t_{0} \in \mathfrak{D}$, then (95) has the property $(\mathrm{P})$ for all $t \in \mathfrak{D}$, and if $(\mathrm{H})$ holds for one $t_{0} \in \mathfrak{D}$, then (95) has the property $(\mathrm{H})$ for all $t \in \mathfrak{D}$.

Finally, we apply the results of this section to prove that the classical as well as the monodromy eigenvalues of the Chandrasekhar-Page angular equation in dependence of $(\mu, \nu) \in \mathbb{R}^{2}$ are (locally) solutions of the partial differential equation (29).

Lemma 8 Let $\kappa \in\left[\frac{1}{2}, \infty\right)$ and $\sigma \in\{-1,+1\}$ be fixed. Moreover, suppose that the functions $v: \mathfrak{D} \longrightarrow \mathbb{R} \backslash\{0\}$ and $w: \mathfrak{D} \longrightarrow \mathbb{R}$ are solutions of the system (50) - (51) on some interval $\mathfrak{D} \subset(0, \infty)$. Finally, let

$$
\mu(t):=\frac{t}{2}\left(v(t)+\frac{\sigma}{v(t)}\right), \quad \nu(t):=\frac{t}{2}\left(v(t)-\frac{\sigma}{v(t)}\right), \quad t \in \mathfrak{D},
$$

and $t_{0} \in \mathfrak{D}$. If $w\left(t_{0}\right)$ is an eigenvalue of $A\left(\kappa ; \mu\left(t_{0}\right), \nu\left(t_{0}\right)\right)$, then $w(t)$ is an eigenvalue of $A(\kappa ; \mu(t), \nu(t))$ for each $t \in \mathfrak{D}$. Furthermore, if $\kappa$ is a half-integer and $w\left(t_{0}\right)$ is a monodromy eigenvalue of $A\left(\kappa ; \mu\left(t_{0}\right), \nu\left(t_{0}\right)\right)$, then $w(t)$ is a monodromy eigenvalue of $A(\kappa ; \mu(t), \nu(t))$ for each $t \in \mathfrak{D}$. 
Proof. In terms of (100) and $\lambda(t):=w(t)$, the coefficient matrices (12) of the system (11) take the form

$$
\begin{gathered}
B_{0}(t)=\left(\begin{array}{cc}
-\frac{\kappa}{2}-\frac{1}{4} & \frac{t}{2}\left(v(t)+\frac{\sigma}{v(t)}\right)-w(t) \\
0 & \frac{\kappa}{2}+\frac{1}{4}
\end{array}\right), \quad B_{1}(t)=\left(\begin{array}{cc}
\frac{\kappa}{2}+\frac{1}{4} & 0 \\
\frac{t}{2}\left(v(t)+\frac{\sigma}{v(t)}\right)-w(t) & -\frac{\kappa}{2}-\frac{1}{4}
\end{array}\right), \\
C(t)=\frac{t}{v(t)}\left(\begin{array}{rr}
-\left(v(t)^{2}+1\right) & -\left(v(t)^{2}-1\right) \\
\left(v(t)^{2}-1\right) & \left(v(t)^{2}+1\right)
\end{array}\right),
\end{gathered}
$$

where the condition (i) is satisfied with $\alpha=-\frac{\kappa}{2}-\frac{1}{4}$ and $\beta=\frac{\kappa}{2}+\frac{1}{4}$. Now, if we define the matrix function

$$
\Omega(x, t):=\frac{1}{v(t)}\left(\begin{array}{cc}
\left(v(t)^{2}-\sigma\right)\left(\frac{1}{2}-x\right) & \left(v(t)^{2}+\sigma\right)(1-x) \\
\left(v(t)^{2}+\sigma\right) x & \left(v(t)^{2}-\sigma\right)\left(x-\frac{1}{2}\right)
\end{array}\right), \quad(x, t) \in \mathbb{C} \times \mathfrak{D},
$$

then, by a straightforward calculation using the characteristic equations (501) - (51), it follows that the deformation equation in (iii) holds. Finally, by setting

$$
G_{0}(t):=\left(\begin{array}{cc}
e^{\phi(t)} & {\left[\frac{t}{2}\left(v(t)+\frac{\sigma}{v(t)}\right)-w(t)\right] e^{-\phi(t)}} \\
0 & \left(\kappa+\frac{1}{2}\right) e^{-\phi(t)}
\end{array}\right), \quad G_{1}(t):=K G_{0}(t) K,
$$

where $K$ is the matrix (20) and

$$
\phi(t):=\int_{t_{0}}^{t} \frac{v(\tau)^{2}-\sigma}{2 v(\tau)} \mathrm{d} \tau, \quad t \in \mathfrak{D},
$$

the conditions (ii) and (iv) are satisfied. Since a point $w(t)$ is a monodromy eigenvalue of $A(\kappa ; \mu(t), \nu(t))$ if and only if (11) has the property (P), and $w(t)$ is a classical eigenvalue of $A(\kappa ; \mu(t), \nu(t))$ if and only if (11) has the property $(\mathrm{H})$, the assertion follows from Corollary 2

Theorem 5 For a fixed $\kappa=k-\frac{1}{2}$ with a positive integer $k$, let $(0,0) \in \mathfrak{S} \subset \mathbb{R}^{2}$ be a simply connected domain such that for each $(\mu, \nu) \in \mathfrak{S}$ all monodromy eigenvalues $\lambda_{0}^{j}(\kappa ; \mu, \nu), j=1-k, \ldots, k-1$, of $A(\kappa ; \mu, \nu)$ are simple zeros of the polynomial $P(\kappa ; \cdot, \mu, \nu)$ given by Theorem 3 Then each function $\lambda=\lambda_{0}^{j}, j=1-k, \ldots, k-1$, satisfies the partial differential equation (29) in $\mathfrak{S}$.

Proof. Let $j \in\{1-k, \ldots, k-1\}$ be fixed. The monodromy eigenvalues of $A(\kappa ; \mu, \nu)$ are exactly the zeros of the polynomial $P(\kappa ; \cdot ; \mu, \nu)$, and since all zeros of $P(\kappa ; \cdot ; \mu, \nu)$ are simple, the implicit function theorem implies that $\lambda_{0}^{j}(\kappa ; \mu, \nu)$ depends analytically on $(\mu, \nu)$ in $\mathfrak{S}$. In order to show that the function $\lambda=\lambda_{0}^{j}$ satisfies the PDE (29), we make use of the unique continuation property of analytical functions. That means, it suffices to prove that (29) holds for $\lambda=\lambda_{0}^{j}$ in a neighbourhood of some point $(\mu, \nu)=(\tau, 0) \in \mathfrak{S}, \tau>0$. Now, in view of the coordinate transformation (100), we have to verify that the function $\lambda_{0}^{j}(\kappa ; \mu(t, v), \nu(t, v))$ is a solution of the partial differential equation (49) in a neighbourhood of the point $(t, v)=(\tau, 1)$. To this end, let us consider the characteristic equations of (49)

$$
\frac{\partial v}{\partial t}(t, u)=-\frac{2 v(t, u) w(t, u)}{t}, \quad \frac{\partial w}{\partial t}(t, u)=-\kappa\left(v(t, u)+\frac{1}{v(t, u)}\right)-\frac{t}{2}\left(v(t, u)^{2}-\frac{1}{v(t, u)^{2}}\right)
$$

together with the initial values

$$
v(\tau, u)=u, \quad w(\tau, u)=\lambda_{0}^{j}(\kappa ; \mu(\tau, u), \nu(\tau, u)),
$$

which depend analytically on the parameter $u \in(0, \infty)$. The solutions $v(t, u)$ and $w(t, u)$ of this initial value problem are analytical functions in a neighbourhood of $(\tau, 1)$, and since $\frac{\partial v}{\partial u}(\tau, u)=1$, they form locally an integral surface for the PDE (49) (compare 12, Chap. 1, Sec. 5]). More precisely, there exists an analytical function $U$ defined on a neighbourhood $\mathfrak{V}$ of $(t, v)=(\tau, 1)$ such that $U(\tau, v)=v$, and $W(t, v):=w(t, U(t, v))$ is a solution of (49) in $\mathfrak{V}$. Now, Lemma 8 implies that $W(t, v)$ is a monodromy eigenvalue of $A(\kappa ; \mu(t, v), \nu(t, v))$ for all $(t, v) \in \mathfrak{V}$, and since $W(\tau, v)=\lambda_{0}^{j}(\kappa ; \mu(\tau, v), \nu(\tau, v))$, it follows that $W(t, v)=\lambda_{0}^{j}(\kappa ; \mu(t, v), \nu(t, v))$ holds identically on $\mathfrak{V}$. This completes the proof of the Theorem. 
In a similar way we can apply Lemma 8 to prove that for fixed $\kappa \in(0, \infty)$ the zeros of the function $\lambda \longmapsto \Delta(\kappa ; \lambda, \mu, \nu)$ defined in Section II and therefore the eigenvalues of $A(\kappa ; \mu, \nu)$ satisfy the partial differential equation (29). This alternative proof of Theorem [1 is based on monodromy preserving deformation - a general technique, which should be applicable to other eigenvalue problems as well. Potential candidates and associated $\Omega$-matrices for solving the deformation equations can be found in [10, Appendix C].

Finally, as a consequence of Theorem [5] the zeros of the polynomial $P(\kappa ; \cdot ; \mu, \nu)$ given by Theorem 3 satisfy the PDE (29) and do not coincide with any eigenvalue of $A(\kappa ; \mu, \nu)$ in a neighbourhood of $(\mu, \nu)=(0,0)$. Moreover (see the proof of Lemma 4), $P(\kappa ; \cdot ; \mu, \nu)$ gives rise to a special integral of polynomial type for the Painlevé III (52). Now, the results of Mansfield \& Webster in [16, Section 2] suggest that these special integrals are unique in some sense, which in turn implies that classical eigenvalues of the Chandrasekhar-Page angular equation are not algebraic.

\section{Appendix}

\section{A. Eigenvalues and eigenfunctions in the case $\mu=\nu=0$}

For fixed $\kappa \in\left[\frac{1}{2}, \infty\right)$, a point $\lambda \in \mathbb{C}$ is an eigenvalue of $A(\kappa ; 0,0)$ if and only if the system (5) with $(\mu, \nu)=(0,0)$ has a nontrivial solution $S(\theta)$ satisfying

$$
\int_{0}^{\pi}|S(\theta)|^{2} \mathrm{~d} \theta<\infty
$$

Introducing the functions $u, v:(-1,1) \longrightarrow \mathbb{C}$ by

$$
S(\theta)=: \sin ^{\kappa+\frac{1}{2}} \theta\left(\begin{array}{l}
\sqrt{\tan \frac{\theta}{2}} u(\cos \theta) \\
\sqrt{\cot \frac{\theta}{2}} v(\cos \theta)
\end{array}\right),
$$

then (15) with $(\mu, \nu)=(0,0)$ is transformed into

$$
(1-x) u^{\prime}(x)=\left(\kappa+\frac{1}{2}\right) u(x)+\lambda v(x), \quad(1+x) v^{\prime}(x)=-\lambda u(x)-\left(\kappa+\frac{1}{2}\right) v(x),
$$

and the normalisation condition (101) is equivalent to

$$
\int_{-1}^{1} u(x)^{2}(1-x)^{\kappa+\frac{1}{2}}(1+x)^{\kappa-\frac{1}{2}} \mathrm{~d} x<\infty, \quad \int_{-1}^{1} v(x)^{2}(1-x)^{\kappa-\frac{1}{2}}(1+x)^{\kappa+\frac{1}{2}} \mathrm{~d} x<\infty .
$$

If $\lambda=0$, then the differential equations (103) imply $u(x)=c_{1}(1-x)^{-\kappa-\frac{1}{2}}$ and $v(x)=c_{2}(1+x)^{-\kappa-\frac{1}{2}}$ with some constants $c_{1}, c_{2} \in \mathbb{C}$, and from the condition (104) it follows that $c_{1}=c_{2}=0$. Hence, $\lambda=0$ is not an eigenvalue of $A(\kappa ; 0,0)$, and we assume in what follows that $\lambda \neq 0$. In this case, the second equation in (103) gives

$$
u(x)=-\frac{1+x}{\lambda} v^{\prime}(x)-\frac{\kappa+\frac{1}{2}}{\lambda} v(x),
$$

and for $v$ we obtain the second order differential equation

$$
\left(1-x^{2}\right) v^{\prime \prime}(x)+[1-2(\kappa+1) x] v^{\prime}(x)+\left[\lambda^{2}-\left(\kappa+\frac{1}{2}\right)^{2}\right] v(x)=0 .
$$

If we set $\alpha:=\kappa-\frac{1}{2}, \beta:=\kappa+\frac{1}{2}$ and $\Lambda:=\lambda-\kappa-\frac{1}{2}$, this differential equation becomes

$$
\left(1-x^{2}\right) v^{\prime \prime}(x)+[\beta-\alpha-(\alpha+\beta+2) x] v^{\prime}(x)+\Lambda(\Lambda+\alpha+\beta+1) v(x)=0,
$$

and the second condition in (104) takes the form

$$
\int_{-1}^{1} v(x)^{2}(1-x)^{\alpha}(1+x)^{\beta} \mathrm{d} x<\infty .
$$


Note that (106) and (107) is the eigenvalue problem associated to the Jacobi polynomials. More precisely, the solutions of the differential equation (106) which are square integrable with respect to the weight function $(1-x)^{\alpha}(1+x)^{\beta}$ are constant multiples of the Jacobi Polynomials $P_{n}^{(\alpha, \beta)}$ with some non-negative integer $n$, and the corresponding eigenvalues $\lambda_{n}^{ \pm}$are determined by the equation $\lambda^{2}-\left(\kappa+\frac{1}{2}\right)^{2}=n(n+\alpha+\beta+1)$, i.e., $\lambda_{n}^{ \pm}= \pm\left(\kappa+\frac{1}{2}+n\right)$. Now, if we define $v(x):=-P_{n}^{(\alpha, \beta)}(x), x \in(-1,1)$, then 105 yields

$$
\begin{aligned}
\lambda_{n}^{ \pm} u(x) & =(1+x) \frac{\mathrm{d}}{\mathrm{d} x} P_{n}^{(\alpha, \beta)}+\beta P_{n}^{(\alpha, \beta)}=\frac{\alpha+\beta+n+1}{2}(1+x) P_{n-1}^{(\alpha+1, \beta+1)}+\beta P_{n}^{(\alpha, \beta)} \\
& =\frac{\alpha+\beta+n+1}{\alpha+\beta+2 n+1}\left[(\beta+n) P_{n-1}^{(\alpha+1, \beta)}+n P_{n}^{(\alpha+1, \beta)}\right]+\beta P_{n}^{(\alpha, \beta)} \\
& =(\alpha+\beta+n+1) P_{n}^{(\alpha+1, \beta)}-(\alpha+n+1) P_{n}^{(\alpha, \beta)}=(\beta+n) P_{n}^{(\alpha+1, \beta-1)}=\left|\lambda_{n}^{ \pm}\right| P_{n}^{(\alpha+1, \beta-1)}
\end{aligned}
$$

where we applied the differentiation formulas and contiguous relations for Jacobi polynomials (see [15, Section 5.2]). Hence, $u(x)= \pm P_{n}^{(\alpha+1, \beta-1)}(x), x \in(-1,1)$, and since $u$ satisfies the first condition in (104), the numbers $\lambda_{n}^{ \pm}$are in fact eigenvalues of $A(\kappa ; 0,0)$. Moreover, the corresponding eigenfunctions are constant multiples of

$$
\sin ^{\kappa} \theta\left(\begin{array}{c} 
\pm \sqrt{\tan \frac{\theta}{2}} P_{n}^{\left(\kappa+\frac{1}{2}, \kappa-\frac{1}{2}\right)}(\cos \theta) \\
-\sqrt{\cot \frac{\theta}{2}} P_{n}^{\left(\kappa-\frac{1}{2}, \kappa+\frac{1}{2}\right)}(\cos \theta)
\end{array}\right), \quad \theta \in(0, \pi),
$$

which form a complete orthogonal set in $\mathscr{L}^{2}\left((0, \pi), \mathbb{C}^{2}\right)$. In particular, the spectrum of $A(\kappa ; 0,0)$ is given by $\left\{\lambda_{n}^{ \pm}\right.$: $n=0,1,2, \ldots\}$.

\section{B. A Numerical Example}

As a numerical example, we have computed the coefficients $c_{m, n}$ of the power series expansion (38) up to and including $m+n=8$ for $\kappa=\frac{1}{2}$ and $j=1$ using the recurrence relation given in Section III. The coefficients have been rounded to six significant figures and listed in the table below. It should be noted that they are to some extent different from the coefficients displayed in [23, Table I]. Evaluating the power series expansion (38) at $\alpha=0.01$ and $\beta=0.02$, i.e., $(\mu, \nu)=(0.005,0.015)$, yields $\tilde{\lambda}_{1}=1.01167$ as a numerical approximation for the eigenvalue $\lambda_{1}$, and this result coincides with the value given in [23, Table II]. For a second pair of parameters $(\alpha, \beta)=(0.5,1.0)$, i.e., $(\mu, \nu)=(0.25,0.75)$, we obtain $\tilde{\lambda}_{1}=1.59745$, which differs slightly from the value $\hat{\lambda}_{1}=1.59764$ listed in $[23$, Table II]. In order to test the reliability of our numerical result, we can use the statement of Lemma 3 That means, we approximate $\Theta(\lambda)$ defined in (25) by the second component $\Theta_{n}(\lambda)$ of $d_{n}(\lambda)$ for $n=8$, and we compare $\Theta_{8}\left(\tilde{\lambda}_{1}\right)$ and $\Theta_{8}\left(\hat{\lambda}_{1}\right)$ with the theoretical result $\Theta\left(\lambda_{1}\right)=0$. As $\Theta_{8}\left(\tilde{\lambda}_{1}\right)=3.60882 \mathrm{e}-05$ and $\Theta_{8}\left(\hat{\lambda}_{1}\right)=-2.51164 \mathrm{e}-04$, our result seems to be more trustworthy. Finally, let $(\mu, \nu)=(0.02,0.1)$. The coefficients of the polynomial $\Theta_{8}$ are given in Table II For these parameters, our power series approximation gives $\tilde{\lambda}_{1}=1.07379$ which differs significantly from

\begin{tabular}{|c|c|c|c|c|c|c|c|c|c|}
\hline & $m=$ & 1 & 2 & 3 & 4 & 5 & 6 & 7 & 8 \\
\hline 2 & $7.40741 e-02$ & $-1.48148 e-02$ & 0.00000 & 0.00000 & 0.00000 & 0.00000 & 0.00000 & & \\
\hline 4 & $-9.14495 e-04$ & $5.48697 e-04$ & $-1.22281 e-04$ & $1.35868 e-05$ & 0.00000 & & & & \\
\hline 5 & $5.08053 e-04$ & $-4.06442 e-04$ & $1.41790 e-04$ & $-2.67091 e-05$ & & & & & \\
\hline 6 & $-3.38702 e-05$ & $3.38702 e-05$ & $-1.63351 e-05$ & & & & & & \\
\hline
\end{tabular}
the value $\hat{\lambda}_{1}=1.06104$ given by Chakrabarti (see [1, Table 1]). Despite his claiming of an accuracy of six decimals, the evaluation of $\Theta_{8}$ at the eigenvalues in question gives $\Theta_{8}\left(\tilde{\lambda}_{1}\right)=5.68899 \mathrm{e}-12$ and $\Theta_{8}\left(\hat{\lambda}_{1}\right)=1.52770 \mathrm{e}-02 \mathrm{in}$ favour of our result. Thus, Chakrabarti's calculations should be taken with some caution.

TABLE I: The coefficients $c_{m, n}, 0 \leq m+n \leq 8$, of the power series expansion 38 in the case $\kappa=\frac{1}{2}$ and $j=1$. 


\begin{tabular}{r|r||r|r}
$n=0$ & $1.22151 \mathrm{e}+00$ & $n=9$ & $4.91151 \mathrm{e}-06$ \\
1 & $1.44347 \mathrm{e}-02$ & 10 & $-4.22048 \mathrm{e}-04$ \\
2 & $-1.70525 \mathrm{e}+00$ & 11 & $-9.46610 \mathrm{e}-08$ \\
3 & $-7.92297 \mathrm{e}-03$ & 12 & $1.02643 \mathrm{e}-05$ \\
4 & $6.72114 \mathrm{e}-01$ & 13 & $6.88933 \mathrm{e}-10$ \\
5 & $1.46003 \mathrm{e}-03$ & 14 & $-1.26470 \mathrm{e}-07$ \\
6 & $-1.12351 \mathrm{e}-01$ & 15 & $-1.00000 \mathrm{e}-26$ \\
7 & $-1.21028 \mathrm{e}-04$ & 16 & $6.15119 \mathrm{e}-10$ \\
8 & $9.39664 \mathrm{e}-03$ & &
\end{tabular}

TABLE II: The coefficients $\delta_{n}$ of the polynomial $\Theta_{8}(\lambda)=\sum_{n=0}^{16} \delta_{n} \lambda^{n}$ for $\kappa=\frac{1}{2}, \mu=0.02, \nu=0.1$.

\section{Eigenfunctions in the case $|\mu| \neq|\nu|$}

Eliminating the second component of $y$ in the system (11), we get a linear second-order differential equation for the first component $y_{1}$ given by

$$
\frac{d^{2} y_{1}}{d x^{2}}(x)+\left(\frac{1}{x}-\frac{1}{x-b}\right) \frac{d y_{1}}{d x}(x)+\left(\tau_{0}+\frac{\tau_{1}}{x}+\frac{\tau_{2}}{x^{2}}+\frac{\tau_{3}}{x-1}+\frac{\tau_{4}}{(x-1)^{2}}+\frac{\tau_{5}}{x-b}\right) y_{1}(x)=0
$$

with

$$
b:=\frac{\mu-\lambda}{2 \mu}, \quad \tau_{0}:=4\left(\mu^{2}-\nu^{2}\right), \quad \tau_{1}:=\lambda^{2}-2 \alpha^{2}+2 \nu+\alpha-\mu^{2}-4 \alpha \nu+\frac{2 \alpha \mu}{\mu-\lambda}, \quad \alpha:=\frac{\kappa}{2}+\frac{1}{4}
$$

and

$$
\tau_{2}:=-\alpha^{2}, \quad \tau_{3}:=\frac{4 \alpha \mu^{2}}{\mu^{2}-\lambda^{2}}+2 \nu-\tau_{1}, \quad \tau_{4}:=\alpha(1-\alpha), \quad \tau_{5}:=\frac{2\left(\nu \mu^{2}+2 \alpha \mu^{2}-\nu \lambda^{2}\right)}{\lambda^{2}-\mu^{2}} .
$$

Now by means of the transformation

$$
y_{1}(x):=x^{\alpha}(x-1)^{\alpha} \psi(x) e^{2 t x}, \quad t= \pm \sqrt{\nu^{2}-\mu^{2}},
$$

we find that $\psi(x)$ satisfies the generalised Heun equation

$$
\frac{d^{2} \psi(x)}{d x^{2}}+\left(\frac{1-\mu_{0}}{x}+\frac{1-\mu_{1}}{x-1}+\frac{1-\mu_{2}}{x-b}+4 t\right) \frac{d \psi(x)}{d x}+\frac{\beta_{0}+\beta_{1} x+\beta_{2} x^{2}}{x(x-1)(x-b)} \psi(x)=0,
$$

where

$$
\mu_{0}=-2 \alpha, \quad \mu_{1}=1-2 \alpha, \quad \mu_{2}=2, \quad \beta_{2}:=8 \alpha t
$$

and

$$
\begin{aligned}
& \beta_{1}=\mu^{2}-\lambda^{2}-2 t[b+2 \alpha(1+2 b)]+2 \alpha(\alpha-1)+2 \nu(2 \alpha-b)-\frac{2 \alpha \mu(b-1)}{\lambda+\mu}+\frac{2 \alpha \mu b}{\mu-\lambda}, \\
& \beta_{0}=b\left(\lambda^{2}-\mu^{2}\right)+b\left[2(\nu+t)-4 \alpha(\nu-t)-4 \alpha^{2}\right]+\alpha-\frac{2 \mu \alpha b}{\lambda-\mu} .
\end{aligned}
$$

We observe that 0,1 and $b$ are simple singularities with characteristic exponents $\left(0, \mu_{0}\right),\left(0, \mu_{1}\right)$ and $\left(0, \mu_{2}\right)$ respectively, while $\infty$ is (at most) an irregular singularity of rank 1. To stress the importance of equation (109), it is sufficient to remark that it contains the ellipsoidal wave equation as well as Heun's equation and thus the Mathieu, spheroidal, Lamé, Whittaker-Hill and Ince equations as special cases.

\section{Acknowledgement}

M. Winklmeier gratefully acknowledges the support of the German Research Foundation, DFG, Grant No. TR 368/4-1, and D. Batic is indebted to the financial support of the MPI für Math. i. d. Naturw., Leipzig, Germany. The authors 
also thank Felix Finster, Universität Regensburg, Germany, and Christiane Tretter, Universität Bremen, Germany, for fruitful discussions. Finally, they thank Alexander Kitaev, Steklov Mathematical Institute, St. Petersburg, Russia, for suggestions on literature about Painlevé III.

[1] S. K. Chakrabarti, On mass-dependent spheroidal harmonics of spin one-half, Proc. Roy. Soc. London Ser. A 391 (1984), no. $1800,27-38$.

[2] S. Chandrasekhar, The solution of Dirac's equation in Kerr geometry, Proc. Roy. Soc. London Ser. A 349 (1976), no. $1659,571-575$.

[3] S. Chandrasekhar, The mathematical theory of black holes, Oxford Classic Texts in the Physical Sciences, Clarendon Press, Oxford, 1998.

[4] B. M. McCoy, C. A. Tracy, And T. T. Wu, Painlevé functions of the third kind, J. Math. Phys. 18 (1977), no. 5, 1058-1092.

[5] M. S. P. Eastham, The Asymptotic Solution of Linear Differential Systems. Applications of the Levinson Theorem, London Mathematical Society Monographs (New Series) 4, Oxford University Press, New York, 1989.

[6] F. Gesztesy, K. Unterkofler, and R. Weikard, On a Theorem of Halphen and its Application to Integrable Systems, J. Math. Anal. Appl. 251 (2000), 504-526.

[7] E. L. Ince, Ordinary Differential Equations, Dover Publications Inc., New York, 1956.

[8] K. Iwasaki, H. Kimura, S. Shimomura, And M. Yoshida, From Gauss to Painlevé: A modern theory of special functions, Aspects of Mathematics E 16, Vieweg Verlag, Braunschweig, 1991.

[9] M. Jimbo, T. Miwa, And K. Ueno, Monodromy preserving deformation of linear ordinary differential equations with rational coefficients. I. General theory and $\tau$-function, Phys. D 2 (1981), no. 2, 306-352.

[10] M. Jimbo, T. Miwa, And K. UEno, Monodromy preserving deformation of linear ordinary differential equations with rational coefficients. II., Phys. D 2 (1981), no. 3, 407-448.

[11] M. Jimbo AND T. MiwA, Monodromy preserving deformation of linear ordinary differential equations with rational coefficients. III, Phys. D 4 (1981/82), no. 1, 26-46.

[12] F. John, Partial differential equations, Fourth edition, Springer, New York, 1982.

[13] E. G. Kalnins And W. Miller, JR., Series solutions for the Dirac equation in Kerr-Newman space-time, J. Math. Phys. 33 (1992), no. 1, 286-296.

[14] T. Kato, Perturbation theory for linear operators, Springer, Berlin - Heidelberg - New York, 1966.

[15] E. Magnus, F. Oberhettinger, And R. P. Soni, Formulas and Theorems for the Special Functions of Mathematical Physics, Springer, Berlin - Heidelberg - New York, 1966.

[16] E. L. Mansfield And H. N. Webster, On One-Parameter Families of Painlevé III, Stud. Appl. Math. 101 (1998), no. $3,321-341$.

[17] J. Meixner And F. W. SchäFke, Mathieusche Funktionen und Sphäroidfunktionen, Springer, Berlin - Göttingen Heidelberg, 1954.

[18] A. E. Milne, P. A. Clarkson, And A. E. Bassom, Bäcklund Transformations and Solution Hierarchies for the Third Painlevé Equation, Stud. Appl. Math. 98 (1997), no. 2, 139-194.

[19] D. PAGE, Dirac equation around a charged, rotating black hole, Phys. Rev. D 14 (1976), p. 1509.

[20] F. W. SснӓFKe, Zur Parameterabhängigkeit bei gewöhnlichen Differentialgleichungen mit singulären Stellen der Bestimmtheit, Math. Nachr. 6 (1951), 45-50.

[21] R. ScHÄFKE, The connection problem for two neighboring regular singular points of general linear complex ordinary differential equations, SIAM J. Math. Anal. 11 (1980), no. 5, 863-875.

[22] R. SCHÄFKE AND D. SCHMIDT, The connection problem for general linear ordinary differential equations at two regular singular points with applications in the theory of special functions, SIAM J. Math. Anal. 11 (1980), no. 5, 848-862.

[23] K. G. Suffern, E. D. Fackerell, And C. M. Cosgrove, Eigenvalues of the Chandrasekhar-Page angular functions, J. Math. Phys. 24 (1983), no. 5, 1350-1358.

[24] W. Wasow, Asymptotic Expansions for Ordinary Differential Equations, John Wiley \& Sons, New York - London Sydney, 1965.

[25] J. Weidmann, Spectral Theory of Ordinary Differential Operators, Lecture Notes in Mathematics 1258, Springer, Berlin - New York, 1987.

[26] H. Widom, On the Solution of a Painlevé III Equation, Math. Phys. Anal. Geom. 3 (2000), no. 4, 375-384. 